\title{
Genome-wide identification of DNA methylation QTLs in whole blood highlights pathways for cardiovascular disease
}

Tianxiao Huan1,2,15, Roby Joehanes, 2,15, Ci Song1,2,3,4, Fen Peng5, Yichen Guo (1) 6,7, Michael Mendelson 1,2,8, Chen Yao (1) 1,2, Chunyu Liu9 ${ }^{9}$, Jiantao Ma1,2, Melissa Richard (1) 5, Golareh Agha ${ }^{10}$, Weihua Guan ${ }^{11}$, Lynn M. Almli (10 ${ }^{12}$, Karen N. Conneely ${ }^{13}$, Joshua Keefe ${ }^{1,2}$, Shih-Jen Hwang ${ }^{1,2}$, Andrew D. Johnson ${ }^{1,2}$, Myriam Fornage ${ }^{5}$, Liming Liang (1) ${ }^{7,14}$ \& Daniel Levy ${ }^{1,2}$

Identifying methylation quantitative trait loci (meQTLs) and integrating them with diseaseassociated variants from genome-wide association studies (GWAS) may illuminate functional mechanisms underlying genetic variant-disease associations. Here, we perform GWAS of $>415$ thousand $\mathrm{CpG}$ methylation sites in whole blood from 4170 individuals and map 4.7 million cis- and 630 thousand trans-meQTL variants targeting >120 thousand CpGs. Independent replication is performed in 1347 participants from two studies. By linking cis-meQTL variants with GWAS results for cardiovascular disease (CVD) traits, we identify 92 putatively causal CpGs for CVD traits by Mendelian randomization analysis. Further integrating gene expression data reveals evidence of cis CpG-transcript pairs causally linked to CVD. In addition, we identify 22 trans-meQTL hotspots each targeting more than $30 \mathrm{CpGs}$ and find that trans-meQTL hotspots appear to act in cis on expression of nearby transcriptional regulatory genes. Our findings provide a powerful meQTL resource and shed light on DNA methylation involvement in human diseases.

\footnotetext{
${ }^{1}$ The Framingham Heart Study, Framingham, MA, USA. ${ }^{2}$ The Population Sciences Branch, Division of Intramural Research, National Heart, Lung, and Blood Institute, National Institutes of Health, Bethesda, MD, USA. ${ }^{3}$ Department of Medical Sciences, Uppsala University, Uppsala, Sweden. ${ }^{4}$ Department of Immunology, Genetics and Pathology, Uppsala University, Uppsala, Sweden. ${ }^{5}$ Brown Foundation Institute of Molecular Medicine, McGovern Medical School, University of Texas Health Science Center at Houston, Houston, TX, USA. ${ }^{6}$ Department of Environmental Health, Harvard T.H. Chan School of Public Health, Harvard University, Boston, MA, USA. ${ }^{7}$ Department of Biostatistics, Harvard T.H. Chan School of Public Health, Harvard University, Boston, MA, USA. ${ }^{8}$ Department of Cardiology, Boston Children's Hospital, Harvard University, Boston, MA, USA. ${ }^{9}$ Department of Biostatistics, Boston University School of Public Health, Boston, MA, USA. ${ }^{10}$ Mailman School of Public Health, Columbia University, New York City, NY, USA. ${ }^{11}$ Division of Biostatistics, School of Public Health, University of Minnesota, Minneapolis, MN, USA. ${ }^{12}$ Department of Psychiatry and Behavioral Sciences, Emory University School of Medicine, Atlanta, GA, USA. ${ }^{13}$ Department of Human Genetics, Emory University School of Medicine, Atlanta, GA, USA. ${ }^{14}$ Department of Epidemiology, Harvard T.H. Chan School of Public Health, Boston, MA, USA. ${ }^{15}$ These authors contributed equally:Tianxiao Huan, Roby Joehanes. Correspondence and requests for materials should be addressed to T.H. (email: tianxiao.huan@nih.gov) or to L.L. (email: Iliang@hsph.harvard.edu) or to D.L. (email: Levyd@nih.gov)
} 
$\mathrm{D}$ NA methylation (DNAm), the covalent binding of a methyl group to the 5' carbon of cytosine occurring mainly at $\mathrm{CpG}$ dinucleotide sequences in the genome, is an important epigenetic regulatory mechanism, and plays a critical role in the regulation of gene expression ${ }^{1}$. Site-specific DNAm is associated with many complex human diseases and traits $^{2}$. DNAm variability is genetically influenced ${ }^{3,4}$, accrues with human aging ${ }^{5}$, and can be altered by environmental exposures such as smoking ${ }^{6}$ and alcohol consumption ${ }^{7}$. Epigenome-wide association studies (EWAS) have identified differentially methylated CpGs associated with numerous clinical traits. The strongest CpGs identified by EWAS, however, seldom reflect a causal role in disease (i.e., $\mathrm{CpG} \rightarrow$ disease), but rather reflect downstream effects of disease processes on the methylome (i.e., disease $\rightarrow$ $\mathrm{CpG})^{8,9}$. Nevertheless, CpGs can serve as useful biomarkers of disease, and the identification of a subset of CpGs that have causal roles in disease can provide insights into disease etiology and potential therapeutic targets.

Genome-wide association studies (GWAS) have recently identified genetic loci associated with site-specific DNAm of CpGs, known as DNA methylation quantitative trait loci (meQTLs) ${ }^{10-14}$. We hypothesized that identifying meQTL variants and linking them to disease-associated genetic variants from GWAS would pinpoint molecular mechanisms underlying genetic susceptibility to human diseases that are due, at least in part, to altered epigenetic regulation. Additionally, it could help explain the molecular consequences of non-protein-coding, diseaseassociated genetic variants from GWAS.

To this end, we perform genome-wide association testing of genetic variants with whole blood DNAm from 4170 European ancestry (EA) participants in the Framingham Heart Study (FHS) and comprehensively map cis- and trans-meQTLs. External replication is performed in 963 EA participants in the Atherosclerosis Risk in Communities (ARIC) study and 384 AfricanAmerican ancestry (AA) participants in the Grady Trauma Project (GTP). We link cis-meQTL single nucleotide polymorphisms (SNPs) with GWAS results for cardiovascular disease (CVD) and its metabolic risk factors and employ Mendelian randomization (MR) to identify putatively causal CpGs for CVD and its risk factors. We further integrate gene expression, gene expressionassociated CpGs, and gene expression-associated QTLs (eQTLs), to reveal causal genomic regulatory pathways for CVD traits. We also report trans-meQTL hotspots, each targeting 30 or more CpGs and demonstrate their influence on cis transcriptional regulatory genes.

\section{Results}

Heritability of global DNAm in peripheral blood. The clinical characteristics of the 4170 FHS participants in this study are summarized in Supplementary Table 1, including 2648 participants from the FHS offspring cohort (mean age 66 years, 54\% women), and 1522 participants from the FHS third generation cohort (mean age 45 years, 52\% women). Among the study participants, 456 are unrelated individuals, and 3,714 are from 511 families of varying sizes (Supplementary Table 2). Figure 1a displays the distribution of heritability estimates $\left(h_{C p G}^{2}\right)$ for 415,318 CpGs. The average heritability of DNAm across all CpGs was estimated to be $0.09 \pm 0.02$ (mean $\pm \mathrm{SD}$ ) and 105,622 CpGs (25.4\%) were found to have $h_{C p G}^{2}>0.1$ (Supplementary Data 1), $39,090(9.4 \%)$ have $h_{C p G}^{2}>0.3$, and $5416(1.3 \%)$ have $h_{C p G}^{2}>0.6$. Genomic features enrichment analysis revealed that $\mathrm{CpGs}$ with $h_{C P G}^{2}>0.1$ were highly enriched for location in enhancer regions (fold enrichment $=1.24, P<1 \mathrm{E}-16$, hypergeometric test), and depleted in promoter, $0-200$ bases upstream of transcription start

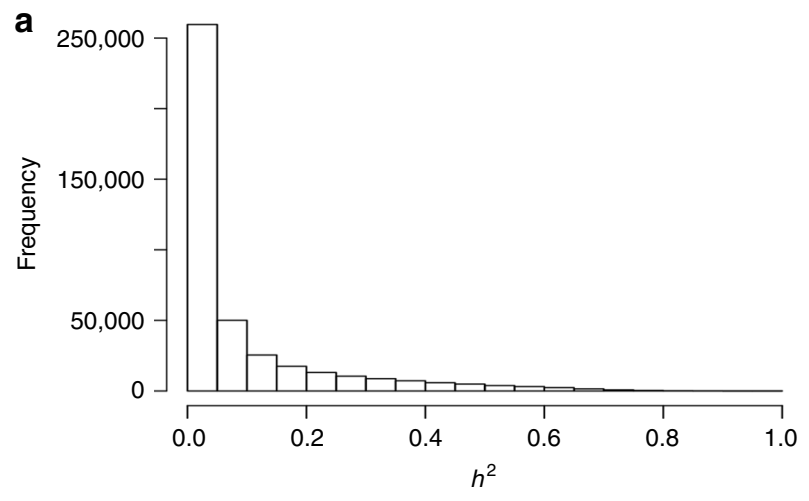

b

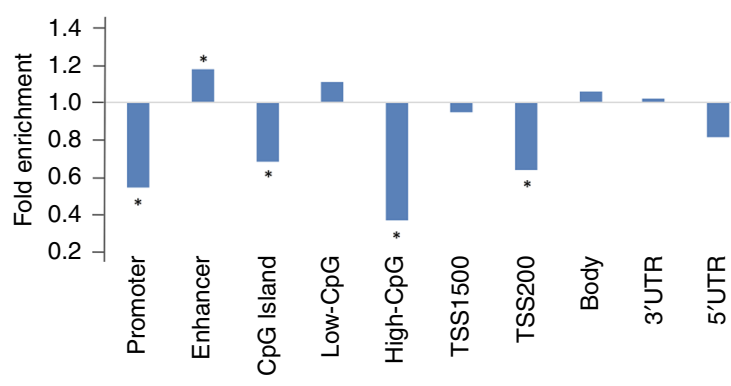

Fig. 1 Heritability analysis of $\mathrm{CpGs}$ genome-wide. a Heritability $\left(h_{C p G}^{2}\right)$ distribution of $415,318 \mathrm{CpGs}$; b enrichment of $\mathrm{CpGs}$ with $h_{\mathrm{CpG}}^{2}>0.1$ in different genomic regions

sites (TSS200), CpG island, and high-CpG dense regions (fold enrichment $<0.8, P<1 \mathrm{E}-16$, hypergeometric test, Fig. $1 \mathrm{~b}$ ). Household effects were positively associated with $h_{C p G}^{2}$, with Pearson correlation $r=0.44$. There were 6,212 CpGs with household effects $>0.1$ (Supplementary Data 2) that showed enrichment for location in 3'UTR regions (fold enrichment $=$ $1.26, P<1 \mathrm{E}-16$, hypergeometric test) and depletion in promoter, TSS200, CpG Islands, and high-CpG dense regions (fold enrichment $<0.8, P<1 \mathrm{E}-16$, hypergeometric test, Supplementary Fig 1).

Identification and replication of cis- and trans-meQTLs. Pairwise association analyses were performed for 8.5 million SNPs and 415 thousand CpGs measured in whole blood samples from 4170 FHS participants. cis-meQTLs were defined as SNPs residing within $1 \mathrm{Mb}$ upstream or downstream of a $\mathrm{CpG}$ site. The distribution of cis-meQTLs in relation to distance from the corresponding $\mathrm{CpGs}$ suggested that a $2 \mathrm{Mb}$ window is a reasonable window for mapping cis-meQTLs (Supplementary Fig 2). We identified 4.7 million cis- (for 121.6 thousand CpGs, 26.8 million pairs) and 706 thousand trans-meQTL SNPs (for 13.5 thousand CpGs, 2 million pairs) at Bonferroni-corrected $P<0.05(P<2 \mathrm{E}$ -11 for cis associations for $2.5 \times 10^{9}$ pairs, and $P<1.5 \mathrm{E}-14$ for trans associations for $3.5 \times 10^{12}$ pairs). Among trans-meQTL SNPs, 33\% reside on the same chromosome as the corresponding CpGs, i.e., intrachromosomal trans-meQTLs, and $67 \%$ were interchromosomal trans-meQTLs. Among intrachromosomal trans-meQTLs SNPs, $70 \%$ reside within $5 \mathrm{Mb}$ of the corresponding CpGs. This indicates that many presumed intrachromosomal trans-meQTLs are not true trans-meQTLs but instead long-range cis-meQTLs. We, therefore, excluded long-range cismeQTLs (i.e., SNPs $>1 \mathrm{Mb}$ but within $5 \mathrm{Mb}$ of $\mathrm{CpGs}$ ), leaving 630 thousand trans-meQTL SNPs (for 10.6 thousand CpGs, 1.6 million pairs). After pruning redundant SNPs at a given locus by limiting linkage disequilibrium [LD] to $r^{2}<0.2$, there remained 
394 thousand independent cis- and 21 thousand transmeQTL SNPs.

Our study, with the largest sample size in a single-site meQTL investigation to date, provides obvious benefits in terms of greater statistical power for discovery (Supplementary Fig 3 displays a flowchart for the identification and replication of meQTLs). We did not observe inflation of the genomic control factor $(\lambda=0.93$, Supplementary Fig 4). By overlapping our meQTL SNP-CpG pairs with previously published results identified in whole blood $^{10,11,13}$ (Supplementary Fig 5), we estimated that our meQTL SNP-CpG pairs cover $80-90 \%$ of significant meQTL SNP-CpG pairs reported in two prior studies ${ }^{10,13}$. Because of the much larger sample size in our study, we detected 3.5 times more cis- and 10 times more trans-meQTL SNPs. A total of 6.9 million cis-meQTL SNP-CpG pairs (27\%) and 206 thousand trans- pairs $(13 \%)$ identified in our study were reported in at least one of the previous meQTL studies.

We further validated our meQTLs by performing independent external replication analysis based on 963 EA samples from the ARIC study and 384 AA samples from GTP. We found that more than $99 \%$ of cis- and trans-meQTL SNP-CpG pairs showed consistent allelic direction of effect in relation to methylation in ARIC vs. FHS (Fig. 2a, b), and $81 \%$ of cis- and trans- pairs showed consistent directions of effect in GTP vs. FHS (Fig. 2c, d). Thirty-six percent of cis- and 39\% of transmeQTL SNP-CpG pairs replicated at Bonferroni-corrected $P<$ 0.05 (corrected for the 26.8 million cis- pairs and 2 million trans-pairs), and $91 \%$ of cis and $94 \%$ of trans pairs replicated at nominal $P<0.05$ in ARIC. $10 \%$ of cis- and $6 \%$ of trans- meQTL SNP-CpG pairs from FHS replicated at Bonferroni-corrected
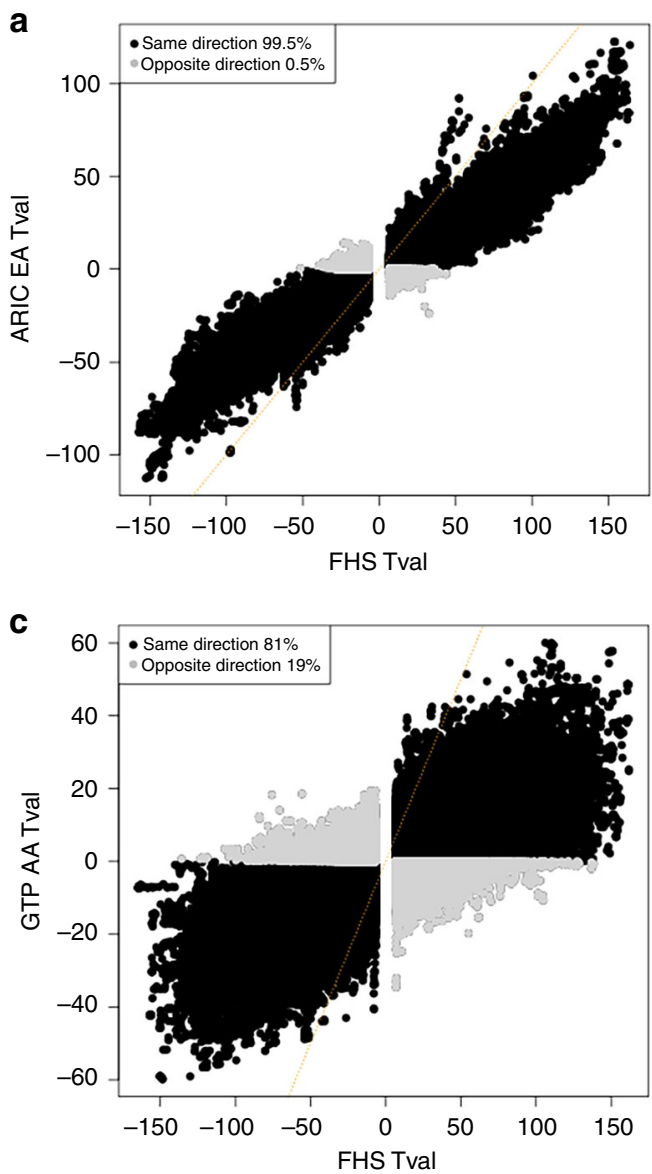

$P<0.05$, and $51 \%$ of cis- and $41 \%$ of trans- pairs replicated at nominal $P<0.05$ in GTP.

Characteristics of cis- and trans-meQTLs. Figure 3 a shows that CpGs with higher heritability $\left(h_{C p G}^{2}\right)$ are more likely to be associated with cis- and trans-meQTL SNPs. Among the CpGs with $h_{C p G}^{2}>0.1,73 \%$ have at least one cis-meQTL SNP, and 7\% have at least one trans-meQTL SNP. Among the CpGs with $h_{C p G}^{2}>0.6$, $76 \%$ have at least one cis-meQTL SNP, and $8 \%$ have at least one trans-meQTL SNP. The mean $( \pm \mathrm{SD})$ proportions of interindividual variation in CpGs explained by the most significant single cis-meQTL SNP $\left(h_{c i s-m e Q T L}^{2}\right)$ is $0.08 \pm 0.10$ and for the most significant single trans-meQTL SNP $\left(h_{\text {trans-meQTL }}^{2}\right)$ it is $0.05 \pm$ 0.06. The proportions of inter-individual variation in $\mathrm{CpGs}$ explained by all of its corresponding cis-meQTL SNPs in aggregate $\left(h_{\text {cis-meQTLs }}^{2}\right)$ is $0.22 \pm 0.25$ and by all of its corresponding trans-meQTL SNPs $\left(h_{\text {trans-meQTLs }}^{2}\right)$ is $0.18 \pm 0.21$ (Fig. 3b). $h_{\text {cis-meQTLs }}^{2}$ and $h_{\text {trans-meQTLs }}^{2}$ are positively correlated with $h_{C p G}^{2}$, with Pearson correlations $r=0.57$ and $r=0.25$, respectively (Fig. 3c). For some meQTLs, $h_{m e Q T L}^{2}$ estimates were large. For example, $h_{m e Q T L}^{2}$ for the cis-meQTL-CpG pair rs62396312-cg03644281 is 0.6. rs62396312 with minor allele frequency $(\mathrm{MAF})=0.11$ is $23 \mathrm{~Kb}$ from cg03644281, which is in the 3'UTR of NFYA (Fig. 3d). $h_{m e Q T L}^{2}$ for trans-meQTL-CpG pair rs2296406-cg04657470 is 0.57. rs2296406 (MAF $=0.38)$ is on Chromosome 16, and cg04657470 is in the first exon of HSPE1 and the 5'UTR of HSPD1 on Chromosome 2 (Fig. 3e).
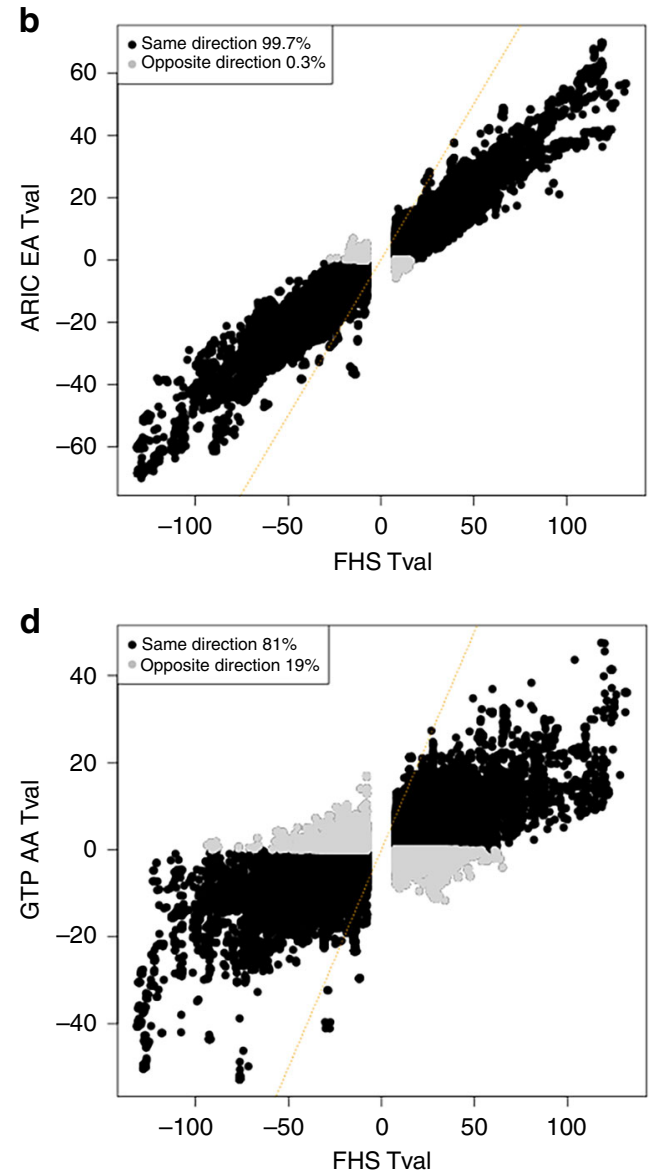

Fig. 2 Plot estimates of cis- and trans-meQTLs in independent studies. a T-values of cis-meQTLs identified in FHS vs ARIC EA; b T-values of trans-meQTLs identified in FHS vs. ARIC EA; c T-values of cis-meQTLs identified in FHS vs GTP AA; d T-values of trans-meQTLs identified in FHS vs. GTP AA 

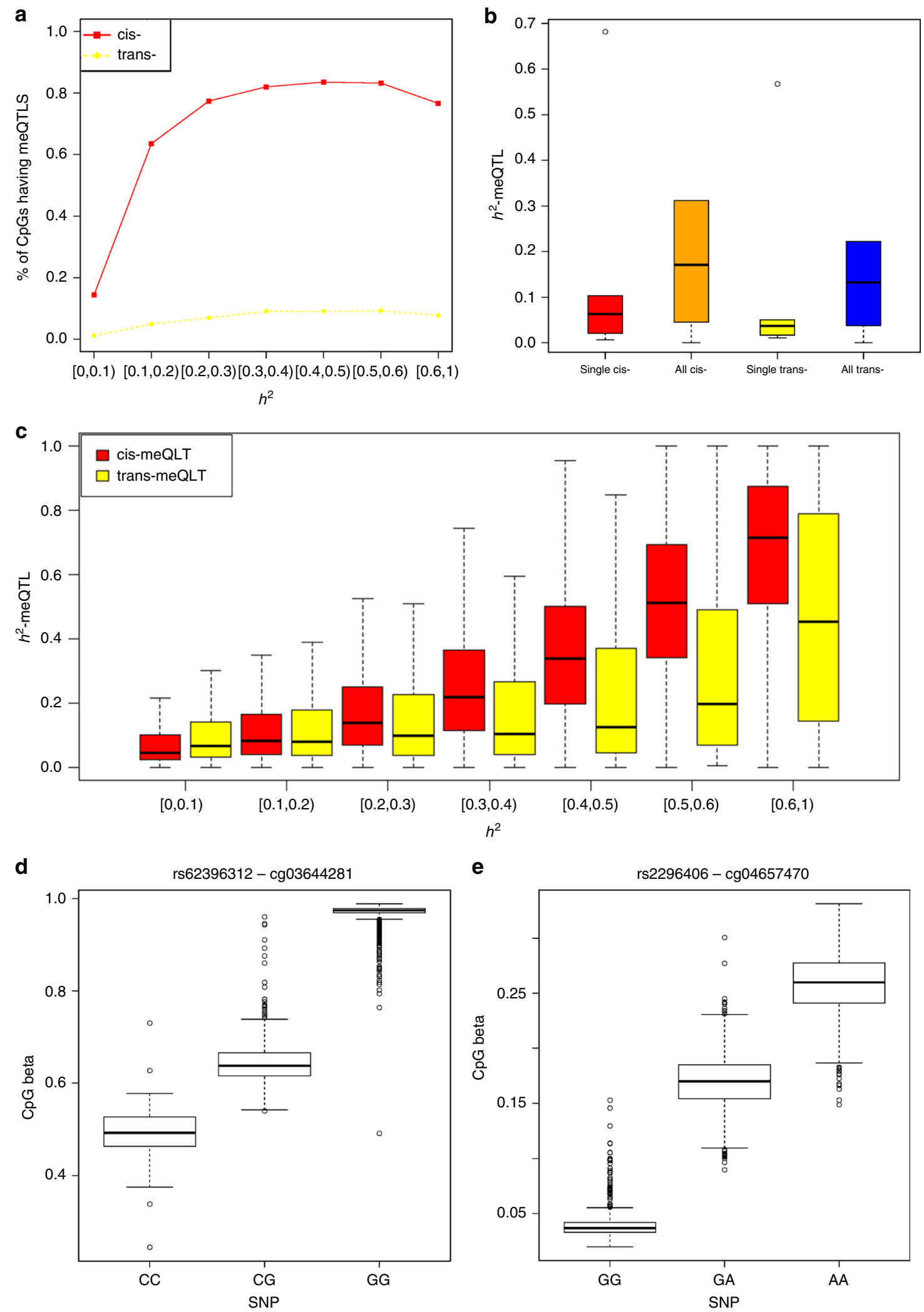

Fig. 3 Characteristics of cis- and trans-meQTLs. a Proportion of CpGs having cis- and trans-meQTL SNPs at different $h_{C p G}^{2}$ levels; b Boxplot summary of $h_{\text {meQTL }}^{2}$ estimated by the peak cis-meQTL SNP, of all cis-meQTL SNPs, the peak trans-meQTL SNP and of all trans-meQTL SNPs. For each CpG, we chose one cis-meQTL SNP with the lowest $P$-value for the CpG as the peak cis-meQTL SNP, and one trans-meQTL SNP with the lowest $P$-value for the CpG as the peak trans-meQTL SNP. c Boxplot summary of $h_{\text {meQTL }}^{2}$ at different $h_{C p G}^{2}$ levels; d Boxplot of the cis-meQTL rs62396312-cg03644281; e Boxplot of the trans-meQTL rs2296406-cg04657470. Boxplots were drawn by the boxplot function in the $R$ library. The boxes indicate the interquartile range (IQR) of values between the 75\% (Q3) and 25\% (Q1). The centre lines indicate the median value. The bars below and above each box indicate the data in Q1-1.5 $x$ IQR and Q3 + 1.5 $x I Q R$, respectively. For $\mathbf{d}, \mathbf{e}, y$-axis shows CpG methylation beta values, and $x$-axis shows SNP genotypes 
Figure 4a shows that cis-meCpGs are enriched in promoter and enhancer regions (fold enrichment $>1.2, P<1 \mathrm{E}-16$, hypergeometric test) and depleted in 5'UTR, TSS200, CpG islands, and high-CpG dense regions (fold enrichment $<0.8$, $P<1 \mathrm{E}-16$, hypergeometric test). In contrast, trans-meCpGs are enriched for high-CpG dense regions (fold enrichment $=$ 1.29, $P<1 \mathrm{E}-16$, hypergeometric test) and depleted in 3'UTR and gene body regions (fold enrichment $<0.8, P<1 \mathrm{E}-16$, hypergeometric test). Overlapping meQTL SNPs with Roadmap project data ${ }^{15}$ measured in primary cells and cell lines from peripheral blood and in many other tissues (see Methods) showed that the cis- and trans-meQTL SNPs are enriched for active chromatin regions, such as transcription start site (TSS) active regions, transcription regions, enhancer regions, and ZNF genes and repeats regions, and highly depleted in heterochromatin and quiescent regions (FDR $<0.05$ based on 1000 permutations; Fig. 4b, c and Supplementary Fig 6). The cis- and trans-meQTL SNPs are also enriched for eQTL SNPs ${ }^{16,17}(P<1 \mathrm{E}-16$, hypergeometric test $)$, protein QTL (pQTLs) SNPs ${ }^{18,19}(P<1 \mathrm{E}-7$, hypergeometric test $)$, and GWAS Catalog $\operatorname{SNPs}^{20}(P<1 \mathrm{E}-16$, hypergeometric test). We found that $87 \%$ of cis-eQTL SNPs, $82 \%$ of cis-pQTL SNPs, and $59 \%$ of GWAS Catalog SNPs are also cis-meQTL variants (Supplementary Table 3).
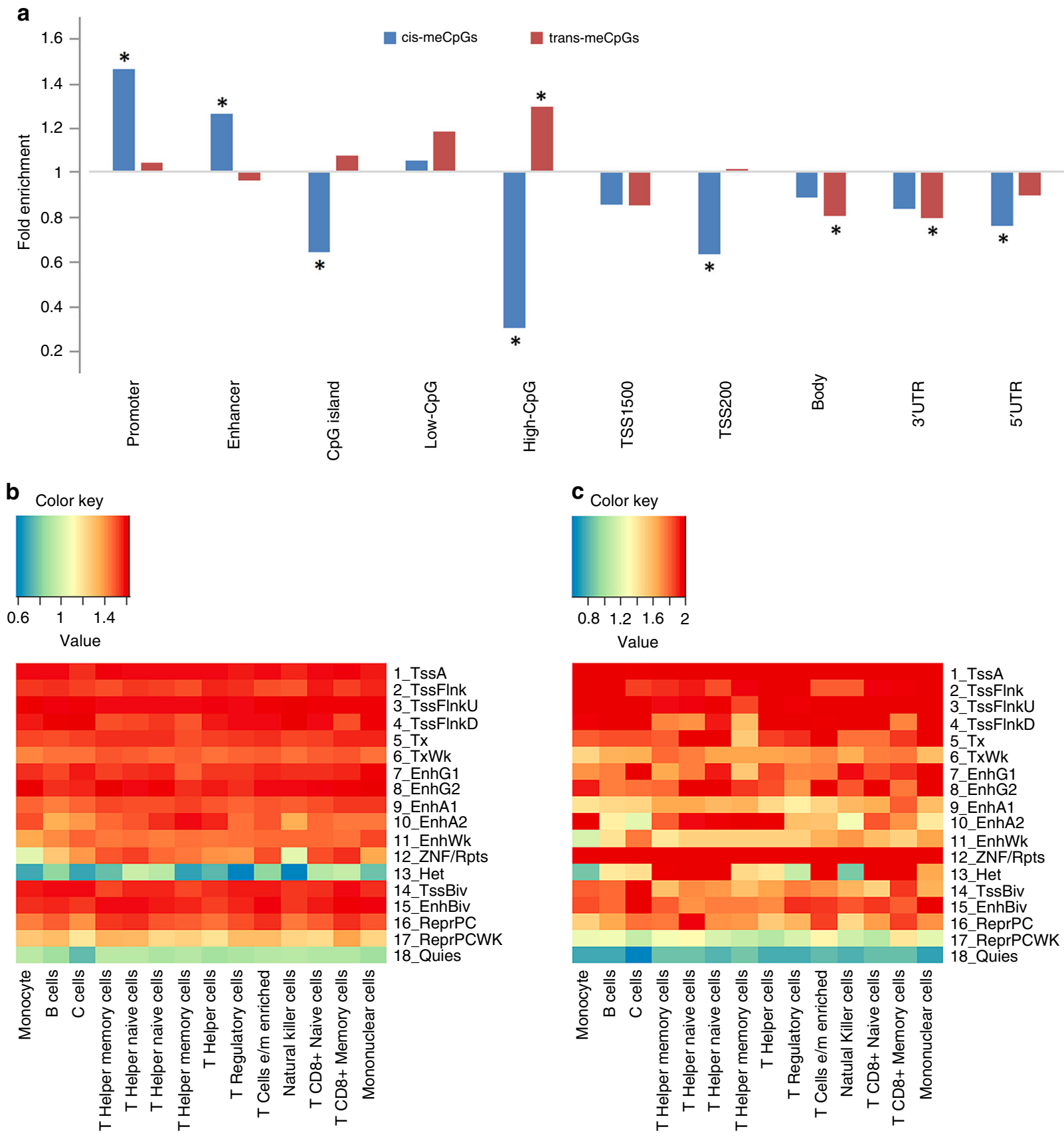

Fig. 4 Genomic features enrichment analysis. a Enrichment of cis- and trans-meQTLs CpGs (meCpGs) in different genomic regions. Red indicated positively associated and green indicated negatively associated. * Indicate significant results with fold change $>1.2$ or $<0.8$, and $P<0.05 / 10$. $\mathbf{b}$ Enrichment of cismeQTL SNPs in different chromatin states in 14 blood cell lines, including monocyte, B cells, $T$ cells, $T$ helper memory cells, $T$ helper naive cells, $T$ helper naive cells, T helper memory cells, T helper cells, T regulatory cells, T cells e/m enriched, Natural killer cells, T CD $8+$ naive cells, T CD $8+$ memory Cells, and mononuclear cells; c Enrichment of trans-meQTL SNPs in different chromatin states 
Using cis-meQTLs to identify causal CpGs for CVD risk. A large proportion (59\%) of GWAS Catalog index SNPs were found to be cis-meQTL SNPs, indicating that a majority of phenotypeassociated SNPs may contribute to disease pathways via effects on local DNAm. We further illustrated in two-sample MR analyses that cis-meQTL variants can be used as instrumental variables (IVs) to identify causal epigenetic mechanisms contributing to CVD and its metabolic risk factors (Fig. 5a). To derive IVs, we identified 159 thousand independent cis-meQTL SNPs pruned by LD $r^{2}<0.01$. By overlapping independent cis-meQTL SNPs with results from GWAS of CVD and its risk factors ${ }^{21-25}$, we identified 14,910 CpGs, each of which has at least three independent cismeQTL SNPs that were suitable instruments for MR to test for causal effects of DNAm on coronary heart disease $(\mathrm{CHD})^{21}$, myocardial infarction $(\mathrm{MI})^{21}$, type-II diabetes (T2D) $)^{22}$, systolic (SBP) and diastolic blood pressure (DBP) ${ }^{25}$, and $9921 \mathrm{CpGs}$ suitable to test causality of DNAm on lipids traits including highdensity lipoprotein (HDL) cholesterol, low-density lipoprotein (LDL) cholesterol, total cholesterol (TC), triglycerides (TG) ${ }^{24}$, and body mass index (BMI ${ }^{23}$.

$\mathrm{CpGs}$ located within $2 \mathrm{Mb}$ and highly correlated with each other $\left(r^{2} \geq 0.5\right)$ shared nearly $100 \%$ of their cis-meQTLs and were considered likely to affect the outcome trait through the same biological mechanism. We, therefore, pruned these CpGs and reported results for the $\mathrm{CpG}$ with the lowest $P$-value in $\mathrm{MR}$ testing as the index or putative causal $\mathrm{CpG}$ for a given window. In contrast, CpGs located close to each other $(<2 \mathrm{Mb})$ but with moderate correlations $\left(0<r^{2}<0.5\right)$ might be more likely to contribute through different regulatory mechanisms and were considered as independent epigenetic sites, even though they shared a partial set of cis-meQTL variants. We used multivariable MR methods to simultaneously estimate the independent causal effect of such CpGs on the outcome.

After correction for multiple testing $(P<0.05 / 14,910$ or $0.05 /$ 9921), MR analysis identified 92 putatively causal CpGs for CVD

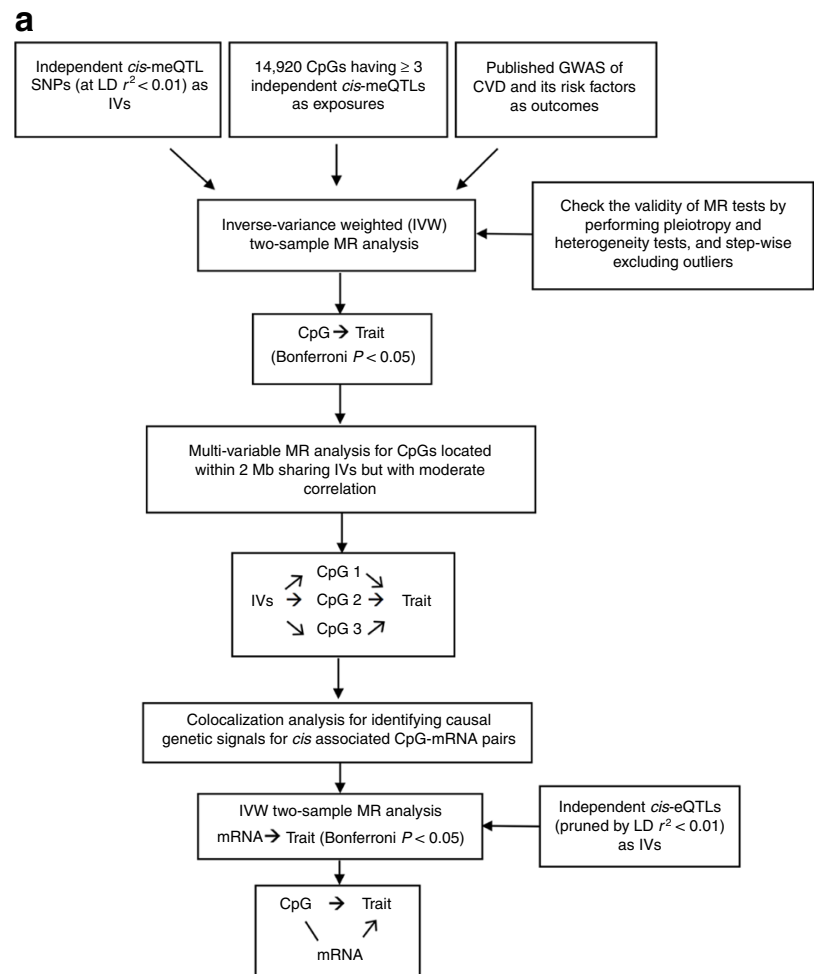

and its risk factors, including 12 putatively causal $\mathrm{CpG}$ for $\mathrm{CHD}$ and MI, four for BMI, 33 for lipids traits, 37 for BP, and eight for T2D. Table 1 shows CHD and MI results and Supplementary Data 3 shows the full list (Supplementary Table 4 shows the multivariable MR results). Among the 12 putatively casual CpGs for $\mathrm{CHD}$ and $\mathrm{MI}$, we found five $\mathrm{CpGs}$ that were positively associated with $\mathrm{CHD} / \mathrm{MI}$ risk (e.g., cg24267699 in $A B O, \mathrm{OR}=$ $\left.2.89, P_{\mathrm{MR}}=1.34 \mathrm{E}-6\right)$, and seven $\mathrm{CpGs}$ inversely associated with $\mathrm{CHD} / \mathrm{MI}$ risk (e.g., cg09803321 in NT5C2, OR $=0.28, P_{\mathrm{MR}}=$ $3.75 \mathrm{E}-15)$. Figure $5 \mathrm{~b}$ lists $30 \mathrm{CpGs}$ that were causal for more than two CVD phenotypes. A striking example is cg16306978 $(A P O B)$, which tested causal (positive direction of effect) for $\mathrm{CHD}\left(\mathrm{OR}=2.46, P_{\mathrm{MR}}=2.1 \mathrm{E}-9\right), \mathrm{LDL}\left(\beta=1.08, P_{\mathrm{MR}}=2.9 \mathrm{E}\right.$ $-24)$, TC $\left(\beta=0.98, P_{\mathrm{MR}}=3.3 \mathrm{E}-22\right)$, and TG $\left(\beta=0.31, P_{\mathrm{MR}}=\right.$ $2.6 \mathrm{E}-6)$. Another example was cg00908766 (CESLR2), which tested causal (positive direction of effect) for $\mathrm{CHD}(\mathrm{OR}=2.18$, $\left.P_{\mathrm{MR}}=4.3 \mathrm{E}-8\right)$ and $\mathrm{MI}\left(\mathrm{OR}=2.01, P_{\mathrm{MR}}=2.3 \mathrm{E}-6\right)$ and inversely causal for HDL $\left(\beta=-0.29, P_{\mathrm{MR}}=2.0 \mathrm{E}-13\right)$.

There were 2951 cis-meQTL SNPs that also were reported to be cis-eQTL variants in GTEx for multiple tissues ${ }^{26}$ (fold enrichment $=1.2, P<1 \mathrm{E}-16$, hypergeometry test). Pathways analysis by FUMA (Functional mapping and annotation of GWAS) ${ }^{27}$ revealed that the cis-meQTLs for the 92 putatively causal CpGs for CVD traits were over-represented with genes involved in sterol metabolic process, regulation of lipoprotein lipase activity, and glycine, serine, and threonine metabolism (Supplementary Data 4).

Identify mRNAs involved in the causal pathways for CVD. We performed comprehensive association analyses of 415 thousand CpGs with expression of $\sim 18,000$ mRNAs measured in 3,684 individuals (Chen Y. et al Unpublished) and found that cis associated CpG-mRNA pairs were replicable in independent external studies, whereas the trans pairs were not. Therefore, in this study, we only focused on mRNAs that were associated in cis

b

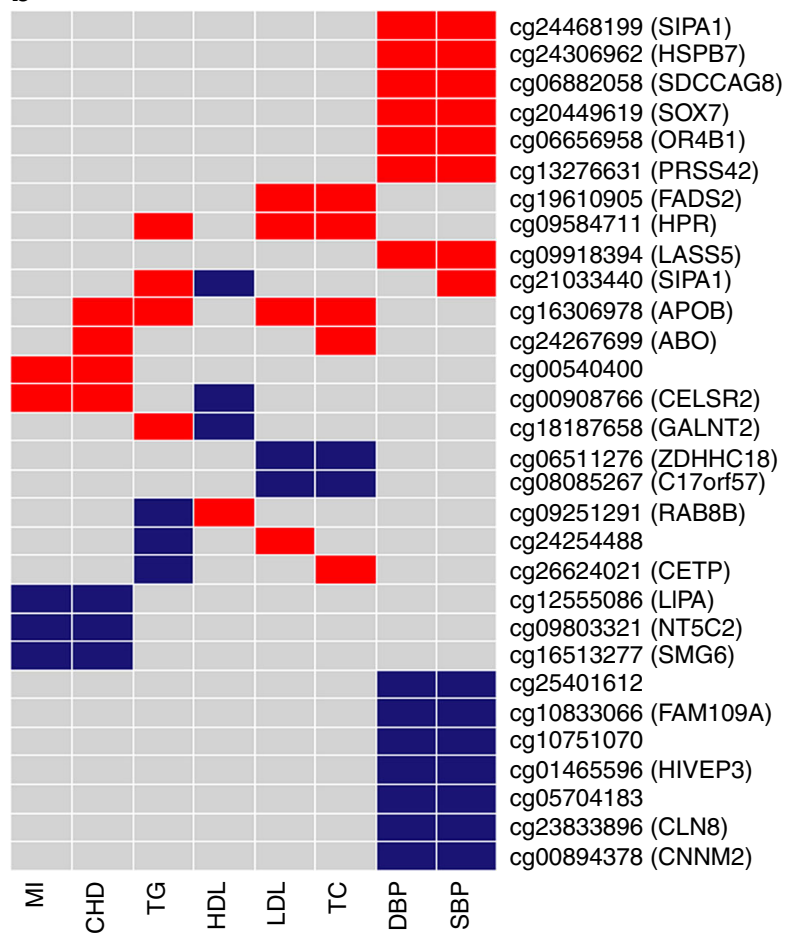

Fig. 5 Mendelian randomization analysis using cis-meQTLs as causal anchors. a Analysis work flow; b Heatmap of 30 CpGs causal for more than two CVD risk factors. The red color shows positive directional effects and the blue color shows negative directional effects 
Table 1 Mendelian randomization results of coronary heart disease and myocardial infarction

\begin{tabular}{|c|c|c|c|c|c|c|c|c|c|c|}
\hline CpG & Phenotype & Chr & Gene & $\begin{array}{l}\text { Number of } \\
\text { independent cis- } \\
\text { meQTLs }\end{array}$ & $\begin{array}{l}\text { IVW MR } \\
\text { test OR }\end{array}$ & $\begin{array}{l}\text { IVW MR } \\
\text { test } 95 \% \mathrm{Cl}\end{array}$ & $\begin{array}{l}\text { IVW MR } \\
\text { test } P \text { - } \\
\text { value }\end{array}$ & $\begin{array}{l}\text { IVW MR test } \\
\text { Bonferroni-corrected } \\
\text { P-value }\end{array}$ & $\begin{array}{l}\text { Heterogeneity } \\
\text { test } \boldsymbol{P} \text {-value }\end{array}$ & $\begin{array}{l}\text { Pleiotropy } \\
\text { test } \boldsymbol{P} \text {-value }\end{array}$ \\
\hline cg09803321 & $\mathrm{CHD} / \mathrm{Ml}$ & 10 & NT5C2 & 3 & 0.28 & $0.20-0.38$ & $3.75 \mathrm{E}-15$ & $5.59 \mathrm{E}-11$ & 0.98 & 0.83 \\
\hline cg12555086 & $\mathrm{CHD} / \mathrm{MI}$ & 10 & LIPA & 4 & 0.42 & $0.32-0.54$ & $1.41 \mathrm{E}-11$ & $2.10 E-07$ & 0.62 & 0.37 \\
\hline cg18534077 & $\mathrm{CHD}$ & 10 & AS3MT & 8 & 0.14 & $0.08-0.26$ & $6.02 \mathrm{E}-11$ & $8.98 \mathrm{E}-07$ & 0.26 & 0.50 \\
\hline cg02493740 & $\mathrm{CHD}$ & 2 & VAMP5 & 7 & 0.33 & $0.23-0.48$ & $1.91 \mathrm{E}-09$ & $2.85 \mathrm{E}-05$ & 0.53 & 0.81 \\
\hline cg16306978 & $\mathrm{CHD}$ & 2 & APOB & 3 & 2.46 & $1.83-3.30$ & $2.09 \mathrm{E}-09$ & $3.12 \mathrm{E}-05$ & 0.73 & 0.48 \\
\hline cg00908766 & $\mathrm{CHD} / \mathrm{MI}$ & 1 & CELSR2 & 5 & 2.18 & $1.66-2.87$ & $4.34 \mathrm{E}-08$ & $6.47 \mathrm{E}-04$ & 0.12 & 0.55 \\
\hline cg00540400 & $\mathrm{CHD} / \mathrm{MI}$ & 15 & & 3 & 3.00 & $2.03-4.45$ & $5.99 E-08$ & $8.93 E-04$ & 0.38 & 0.57 \\
\hline cg16513277 & $\mathrm{CHD} / \mathrm{MI}$ & 17 & SMG6 & 3 & 0.06 & $0.02-0.17$ & $1.99 E-07$ & $2.97 \mathrm{E}-03$ & 0.62 & 0.43 \\
\hline cg21433558 & $\mathrm{CHD}$ & 17 & CNTNAP1 & 5 & 2.69 & $1.82-3.98$ & $6.73 \mathrm{E}-07$ & $1.00 E-02$ & 0.70 & 0.36 \\
\hline cg14037218 & $\mathrm{CHD}$ & 1 & ADAMTSL4 & 3 & 0.14 & $0.07-0.31$ & $1.20 E-06$ & $1.79 \mathrm{E}-02$ & 0.81 & 0.53 \\
\hline cg24267699 & $\mathrm{CHD}$ & 9 & $\mathrm{ABO}$ & 4 & 2.89 & $1.88-4.44$ & $1.34 \mathrm{E}-06$ & $2.00 \mathrm{E}-02$ & 0.61 & 0.47 \\
\hline cg21692620 & $\mathrm{MI}$ & 17 & CNTNAP1 & 4 & 0.26 & $0.15-0.45$ & $1.41 \mathrm{E}-06$ & $2.10 \mathrm{E}-02$ & 0.57 & 0.57 \\
\hline
\end{tabular}

For CpGs that tested causal for both $\mathrm{MI}$ and CHD, only the MR results for CHD are shown in this table. The full MR results are shown in Supplementary Data 3

Bonferroni-corrected $P$-value is corrected for the number of $\mathrm{CpGs}$ having $\geq 3$ independent cis-meQTLs $(N=14,910)$

Independent cis-meQTLs were defined using LD $r^{2}<0.01$

with the $92 \mathrm{CpG}$ that tested positive in MR analyses. Among the 92 CpGs, 26 were associated in cis with 29 mRNAs in 3,684 individuals, including $35 \mathrm{CpG}$-mRNA pairs. Colocalization analysis revealed eight cis-associated CpG-mRNA pairs (including eight CpGs and eight mRNAs) for which DNAm and gene expression changes were driven by the same causal genetic variant, i.e., the causal cis-meQTL variant was also the causal ciseQTL variant with a probability of $>80 \%$.

For these eight mRNAs, we used cis-eQTL SNPs as IVs in MR analysis to test if the expression changes are causal for CVD traits. At $P<0.05 / 8$, we identified five genes whose expression was causal for CVD traits in whole blood using FHS-eQTL variants ${ }^{16}$ (Supplementary Data 5), and five genes reflecting multiple tissues using GTEx eQTL variants ${ }^{26}$ (Supplementary Table 5). Our results show for example that cg12555086, located in the LIPA gene body region, is associated in cis with expression of LIPA $(\beta=-4.4$, and $P=1 \mathrm{E}-277)$. A cis-meQTL variant associated with cg12555086 colocalized with a cis-eQTL variant associated with LIPA expression at a probability $>0.99$. LIPA expression tested causal for both $\mathrm{CHD}\left(\mathrm{OR}=0.42\right.$, and $P_{M R}=$ $1.4 \mathrm{E}-11)$ and $\mathrm{MI}\left(\mathrm{OR}=0.36\right.$, and $\left.P_{M R}=6.1 \mathrm{E}-12\right)$ in whole blood (Fig. 6a, b and Supplementary Fig 7a, b). The expression levels of LIPA were also tested casual for CHD and MI in many other tissues including adrenal gland, aorta, and liver. Another example is cg06882058 located in the gene body region of SDCCAG8; it is associated in cis with expression of SDCCAG8 $(\beta=1.72$, and $P=1 \mathrm{E}-22)$. A cis-meQTL variant associated with cg06882058 colocalized with a cis-eQTL variant associated with expression of SDCCAG8 (colocalization probability $>0.99$ ). SDCCAG8 expression tested causal for both SBP $(\beta=13.46$, and $P_{M R}=7.2 \mathrm{E}-8$, $)$ and DBP $\left(\beta=10.40\right.$, and $\left.P_{M R}=1.6 \mathrm{E}-7\right)$ in whole blood (Fig. 6c, d and Supplementary Fig $7 c, d$ ). The expression levels of SDCCAG8 also tested casual for SBP and DBP in many other tissues including tibial artery, heart atrial appendage, aorta, and brain. These results highlight many putatively causal pathways for CVD traits involving both DNAm and gene expression changes. Further experimental validation is needed to definitively prove causality.

trans-meQTLs target local gene expression. The molecular mechanism underlying trans-meQTLs is unknown. In a previous study, Shi et al reported a SNP (rs12933229) that was associated with five $\mathrm{CpGs}$ residing on different chromosomes ${ }^{12}$. In another study, Bonder et al explored trans-meQTL variants among 6111 GWAS Catalog SNPs and proposed that some genetic variants that affect the activity of a transcription factor in cis were associated in trans with DNAm changes at its binding sites ${ }^{11}$. In the present study, we identified 630 thousand trans-meQTL variants (6 million SNP-CpG pairs) genome-wide (Supplementary Fig 8) and demonstrated that trans-meQTLs are replicable in independent external studies.

Among the 630 thousand trans-meQTL variants that we report, 547 thousand (89\%) also were cis-meQTL variants, and 178 thousand (28\%) were cis-eQTL variants (enrichment test at $P<1 \mathrm{E}$ -16 , hypergeometry test). The multiple roles of meQTLs lead us to hypothesize that SNPs with trans-acting effects on remote CpGs may do so via effects on nearby genes. We detected 22 transmeQTL hotspots (denoted as H1 to H22), defined on the basis of an index SNP associated with at least 30 trans-meCpGs (Supplementary Data 6 and Fig. 7a). The 22 trans-meQTL hotspots targeted a total of 1701 trans-meCpGs. A total of $28 \%$ $(875 / 3077)$ of the trans-meQTL hotspots variants were also ciseQTLs associated with expression levels of 74 nearby genes (ciseGenes). There were 146 cis-eGene-trans-meCpG associated pairs (at multiple testing corrected $P<0.05$, including 19 cis-eGenes linked to 130 trans-meCpGs) in 3684 FHS participants. A hypergeometric test suggested that among all CpGs $(n=901)$ associated with cis-eGenes, there was enrichment for transmeCpGs ( $n=130, P<1 \mathrm{E}-16$, hypergeometry test). Gene ontology enrichment analysis showed that the 74 eGenes were enriched for transcription regulatory genes (24 genes, H2: TCF7L1; H13: ZNF200, ZNF75A; H14: INO80E; H19: ZNF177, ZNF266, ZNF561; H20: ZNF333; H21; ZNF100, ZNF208, ZNF429, ZNF492, ZNF493, ZNF738; H22: HKR1, ZFP30, ZNF260, ZNF540, ZNF566, ZNF573, ZNF585B, ZNF607, ZNF781, ZEN793) and DNA binding genes (22 genes, H3: SP140L; H13: ZNF75A; H17: E2F4; H19: ZNF177, ZNF266; H20: ZNF333; H21; ZNF208, ZNF429, ZNF493; ZNF738; H22: HKR1, ZNF260, ZNF781, ZNF607, ZNF540, ZNF566, ZNF573; Supplementary Table 6). One possible explanation for this observation is that the trans-meQTL SNPs may affect transcription regulatory genes in cis, and these transcription regulatory genes interact with trans-meCpGs to regulate their target genes (Fig. 7b). Further functional experiments are needed to prove this hypothesis.

\section{Discussion}

Using a large single-site community-based cohort, we identified over 4.7 million cis- and 630 thousand trans-meQTL variants, roughly 3.5 times (cis-) and 10 times (trans-) more than the previous studies ${ }^{10,11,13}$. Independent external replication revealed that a large proportion of cis- and trans-meQTLs are replicable and the majority of our meQTL-CpG pairs showed directional concordance in independent external cohorts of participants of EA (99\%) and AA (81\%) 
a

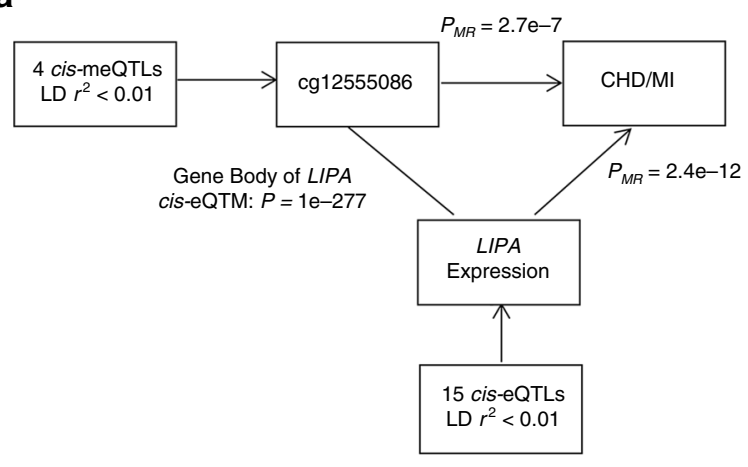

C

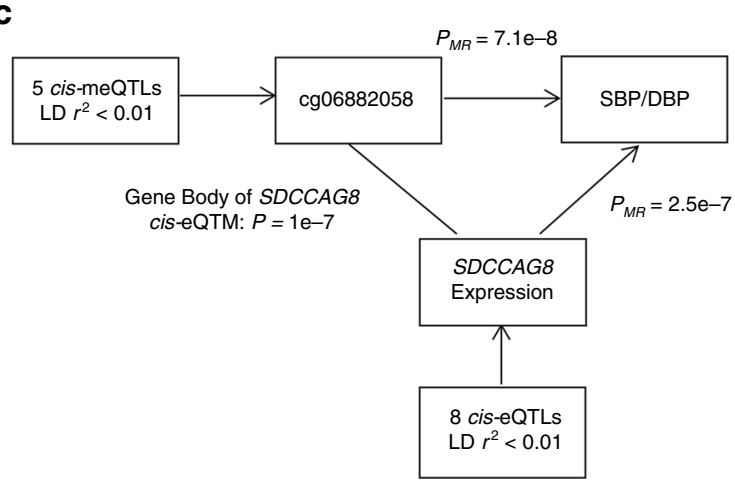

b
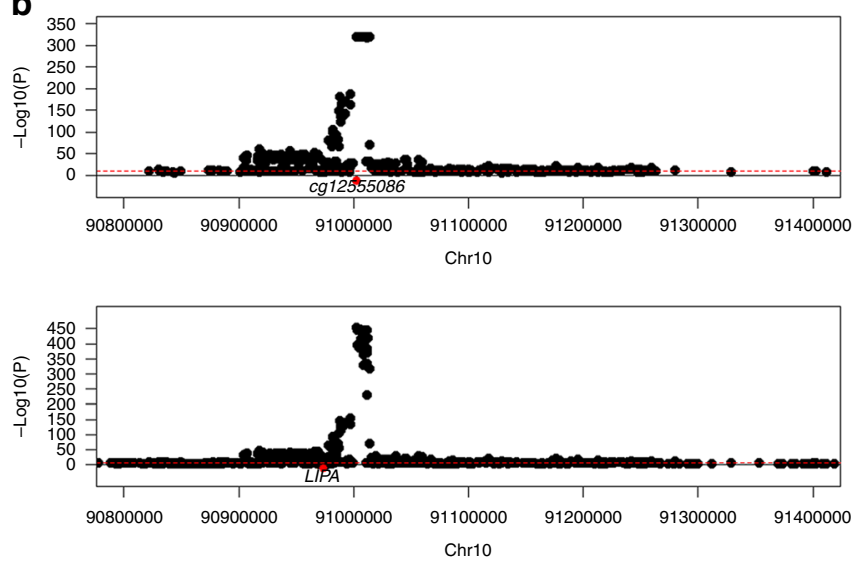

d

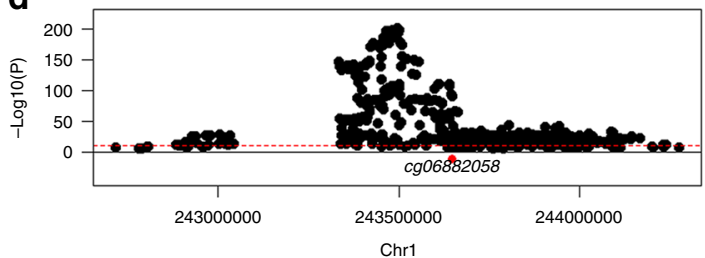

Chr1

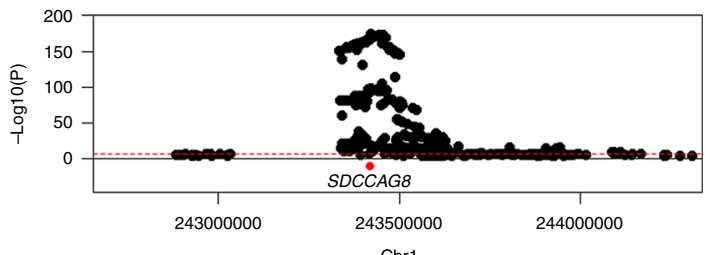

Fig. 6 Mendelian randomization examples. a MR example of cg12555086 in relation to CHD and MI; b colocalization of a casual cis-meQTL and a casual cis-eQTL on cg12555086 and LIPA expression; c MR example of cg06882058 in relation to SBP and DBP; $\mathbf{d}$ colocalization of a casual cis-meQTL and a casual cis-eQTL on cg06882058 and SDCCAG8 expression

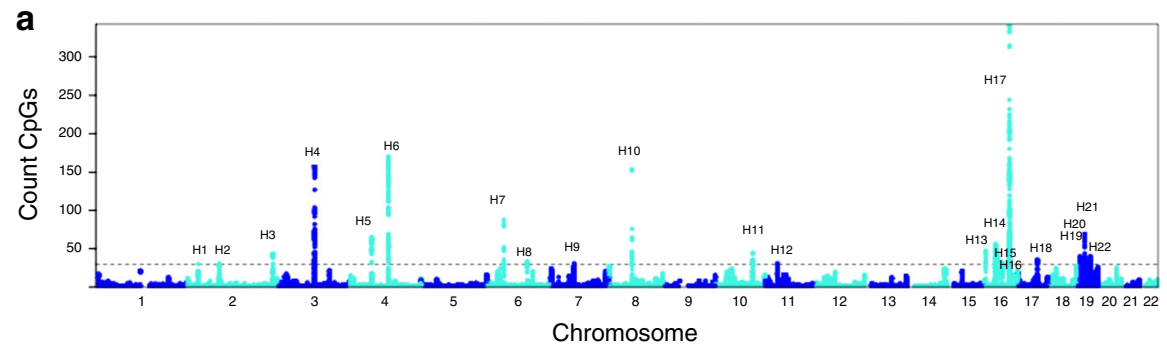

b

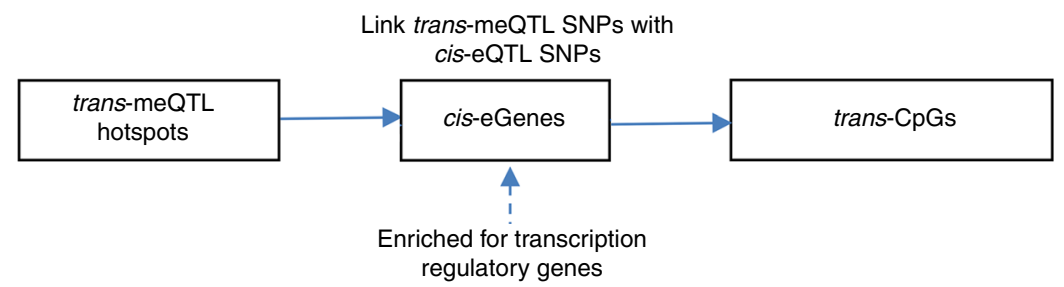

Fig. 7 Overview and characteristics of trans-meQTLs. a trans-meQTL hotspots; b Linking trans-meQTLs with cis-eGenes

ancestry. The comprehensive meQTL resources provided by our study reveal a new richness of detail regarding genetic effects on DNAm patterns and the potential causal relation of epigenetic influences on various disease phenotypes. In this way, we help bridge a GWAS gap regarding diseaseassociated SNPs.
Our study is a well-powered multi-generational study. Thus, we were able to report accurate heritability estimates of DNAm in peripheral blood. We found that the average $h_{C p G}^{2}$ of all 415 thousand CpGs is 0.09 , which is comparable to the values reported in a twins study $(0.18-0.19)^{28}$ and two family-based studies $(0.13-0.14)^{29,30}$. Mapping of meQTLs suggested that 
CpGs with higher $h_{C p G}^{2}$ were more likely to have cis- and transmeQTL SNPs. The proportion of interindividual variation in methylation of CpGs explained by cis- and trans-meQTL SNPs increased proportionally with $h_{C p G}^{2}$.

The meQTLs identified in the FHS show excellent replicability in independent studies. As shown in Fig. 2, meQTLs from the FHS show $99 \%$ concordance in the ARIC EA cohort and $81 \%$ in the GTP AA cohort. Because the sample sizes in ARIC ( $n=$ 963) and GTP $(n=384)$ were much smaller than in the FHS $(n=4170)$, the trend for T values of meQTLs was skewed and reflects the obvious benefits of using a large cohort in terms of greater statistical power to identify novel meQTLs. It is also the primary reason that our study identified many more cis- and trans-meQTLs than previous studies. Our study replicated the majority of meQTL SNP-CpG pairs identified in prior studies $^{10,11,13}$ (Supplementary Fig 5, 87\% of cis- reported by Bonder et al. ${ }^{11}, 91 \%$ of cis- and $89 \%$ trans-pairs reported by Gaunt et al. ${ }^{13}$, and $81 \%$ of trans-pairs reported by Lemire et al. ${ }^{10}$ ). Our study only replicated $36 \%$ of reported peak cis-meQTLs from Lemire et al. This low value might be because the peak cismeQTLs in Lemire et al may not be as robust as others that could be largely replicated in our study. We acknowledge this as a limitation of our study that genotypes were from 1000 Genomes imputation data. Whole-genome sequencing methods for determining true genotypes rather than imputation will offer higher resolution and reduce the imputation uncertainty. Our study only replicated $10 \%$ of trans-meQTLs from Bonder et al, perhaps because they only focused on trans-meQTLs for GWAS Catalog SNPs and used a much lower $P$-value threshold $(P<2.6 \mathrm{E}-7)$ in their study versus $P<1.5 \mathrm{E}-14$ in our study. The failure to replicate the remaining $10-20 \%$ cis- and trans-meQTLs identified in those previous studies might be due to different cohorts, genotype imputation accuracy, different statistical significance thresholds for meQTLs, or hidden confounding in the methylation data. Another limitation in our study is the limited coverage of the Illumina HumanMethylation450 platform, which may result in many missed meQTLs for CpGs that were not measured.

To demonstrate the utility of our meQTL resource, we explored several key CVD-related traits and performed MR to identify examples of CpGs that are putatively causal for CVD, which remains the leading cause of death worldwide ${ }^{31}$. Recently, several EWAS have reported CpGs associated with CVD risk factors ${ }^{8,32-34}$, but the reported associations might not reflect causal effects owing to the inability to exclude possible alternative mechanisms, such as downstream effects of disease on methylation, or unexplained confounding factors. In fact, the vast majority of trait-associated CpGs from EWAS are more likely to reflect effects of the trait on DNA methylation rather than causal effects of methylation on disease ${ }^{8,9,35}$. A large database of meQTL variants enabled us to conduct systematic MR analyses using cismeQTL variants as IVs to identify causal effects of DNAm on CVD traits. A similar recent study by Richardson et $\mathrm{al}^{36}$ reported MR analysis using single cis-meQTL variants as IVs by focusing on $10 \mathrm{CpGs}$ pre-selected based on EWAS and GWAS. Richardson et al acknowledged that the single IV MR method cannot separate causality from horizontal pleiotropy where genetic variants affect the exposure and outcome simultaneously ${ }^{36}$. In our study, because of the large number of meQTLs discovered, we were able to explore the direction of causality for more than 14 thousand candidate CpGs on CVD risk factors using multiple independent cis-meQTL variants $(\geq 3)$ as IVs. In comparison with single-IV MR methods, the multiple-variant IV method increased the proportion of the variance in CVD traits explained versus a single variant and reduced the bias of horizontal pleiotropy that can affect single cis-meQTL MR analysis. Therefore, our approach has greater power to identify causal CpGs. Among all CpGs that tested causal for CVD traits, $24 \%$ have cis-meQTL variants that also are genome-wide significant $(P<5 \mathrm{E}-8)$ in GWAS of CVD traits (Table 1 and Supplementary Data 7). cis-meQTLs for the other causal CpGs were moderately associated with CVD and its risk factors in GWAS.

Some of the causal CpGs identified in this study reside in known CVD-related genes. For example, we identified cg12555086 for LIPA as being causal for CHD and MI. LIPA encodes lipase $A$, which is also known as cholesterol ester hydrolase. This enzyme functions in the lysosome to catalyze the hydrolysis of cholesteryl esters and triglycerides. Loss-of-function mutations in LIPA result in accelerated atherosclerosis ${ }^{37}$. Another example is six CpGs at the $A B O$ locus that we found to be casual for CHD, MI, and total cholesterol levels (Supplementary Data 3). The $A B O$ locus has been reported to be associated with CVD traits in previous studies ${ }^{21,38}$. ABO blood type has previously been linked to CVD risk in the FHS ${ }^{39}$. These published studies support our findings. In addition, our study provides evidence that changing expression levels for these genes may contribute to CVD risk. Some CpGs that tested positive by MR have not previously been reported to play a causal role in CVD. For example, we identified cg06882058 in SDCCAG8 as causal for both SBP and DBP. CpG cg06882058 is associated in cis with expression of SDCCAG8, which we found to be causal for both SBP and DBP (Supplementary Data 5 and Supplementary Table 5). Previous studies found that SDCCAG8 causes nephronophthisis type 10, characterized by retinal and renal degeneration, mild intellectual disability, obesity, hypogonadism, and recurrent respiratory infections in humans ${ }^{40,41}$. Sdccag8 knockout mice develop lateonset nephronophthisis and severely increased $\mathrm{BP}^{42}$. This evidence leads us to hypothesize that dysregulation of cg06882058 may affect expression of SDCCAG8 and thereby cause hypertension and contribute to CVD risk. Further experimental validation is necessary to prove this hypothesis.

There is considerable merit for using meQTLs along with other molecular QTLs, such as eQTLs to reveal much broader and more complex gene networks underlying genetic variant-disease associations. We show in the colocalization analysis that CpGs and their cis-associated gene expression are driven by the same causal variants. This suggests the presence of "vertical causal pathways" linking genetic variants, DNAm, and gene expression to human diseases.

Previous studies have revealed that DNAm patterns are highly tissue specific ${ }^{43}$. An important limitation of our study is that we may not detect many causal CpGs for CVD in whole bloodderived DNA when their contributions to disease is due to altered methylation in other tissues that are relevant to CVD. By overlapping meQTL SNPs with Epigenome Roadmap Project data (Fig. 4 and Supplementary Fig 6), our results show that the cisand trans-meQTLs are enriched in transcription active regions and depleted in heterochromatin and quiescent regions in multiple tissues. It seems plausible that the putatively causal pathways derived by CpGs identified from whole blood are shared across tissues. MR testing utilizing GTEx eQTL variants ${ }^{26}$ further confirms that many mRNAs lying on the CpG-derived pathways for CVD in whole blood were also causal for CVD traits in multiple tissues, including heart, liver, adipose, and others (Supplementary Data 5 and Supplementary Table 5).

Our study did not find causal CpGs among the top reported results of recently published EWAS of $\mathrm{BP}^{34}$, lipids ${ }^{33}$, and $\mathrm{BMI}^{8,32}$. As discussed above, CpGs reported by EWAS are more likely to reflect downstream effects of the trait on DNAm rather than causal effects of DNAm on disease ${ }^{8,9,35}$. Published EWAS may also have limited power to observe associations of causal CpGs with traits, perhaps because causal effects of CpGs on disease are smaller than the effects of disease on methylation of CpGs. One of 
the most promising results of our study is for cg16306978 in $A P O B$, an LDL particle ligand, which tested causal for CHD, LDL, TC, and TG (Supplementary Data 3). APOB protein has been linked to long-term CVD risk ${ }^{18}$. We found 12 of the 92 causal CpGs to be associated with smoking ${ }^{6}$, and six CpGs were associated with alcohol consumption ${ }^{7}$ (at $P<0.05 / 92$; Supplementary Table 7). Our results may bridge a knowledge gap by explaining how environmental influences can alter epigenetic patterns that in turn affect diseases.

Our trans-meQTL results revealed an abundance of transmeQTL hotspots and illustrated their putative activity on proximal nuclear binding genes and transcriptional regulatory genes. Our results are consistent with findings reported by Bonder et al. $^{11}$ and by Lemire et al. ${ }^{10}$. However, the trans-meQTLs reported by Bonder et al were limited to trans-meQTL variants that also are GWAS Catalog $\mathrm{SNPs}^{11}$. Lemire's study identified fewer than 2000 trans-meQTL SNP-CpG pairs due to a smaller sample size ${ }^{36}$. In contrast, we systematically mapped 22 transmeQTLs hotspots that targeted more than $30 \mathrm{CpGs}$ on different chromosomes. We replicated Shi et al.'s trans-meQTL hotspot finding (H14 in our results) ${ }^{12}$. Another promising example in our study, H17 (with a peak SNP rs7203742 located in an intron of CTCF on chromosome 16, Fig. 7a) is cis-acting on 17 genes, including several transcription regulatory genes, E2F4, NUTF2, and NFATC3, targeting 343 CpGs across the genome. rs7203742 was also identified by Lemire et al. as a trans-meQTL targeting 14 $\mathrm{CpGs}^{10}$. We speculate that these transcriptional regulatory genes may either coincide with their trans-associated CpGs to regulate downstream gene expression or involve direct regulation of DNAm of trans-associated CpGs. Further functional experiments are needed to explore molecular mechanisms underlying such trans phenomena. In our previous study, we identified 13 transeQTL hotspots that affected hundreds of genes ${ }^{44}$. Our previously reported trans-eQTLs hotspots did not overlap with the transmeQTL hotspots identified in the present study, and the ciseGenes affected by trans-eQTL hotspots did not show enrichment of transcriptional regulatory genes. Instead, the trans-eQTL hotspots were enriched for platelet SNPs and platelet eQTL variants. This finding indicates that trans-eQTLs are highly tissue specific, in contrast to the trans-meQTLs, which reflect remote control by transcriptional regulatory genes.

\section{Methods \\ Study populations. The discovery study used 4170 EA participants from the FHS, a community-based study of cardiovascular disease and its risk factors ${ }^{45}$. In 1971 , the Offspring Generation cohort was recruited, consisting of the immediate des- cendants (and their spouses) of the Generation 1 cohort $^{46}$. From 2002 to 2005, the Third Generation cohort was recruited, consisting of immediate descendants of the Offspring Generation cohort participants ${ }^{47}$. In this study, eligible participants included participants from the Offspring Generation cohort who attended their eighth examination cycle (Exam 8, 2005-2008, $N=2648$ ), and Third Generation cohort participants who attended their second examination cycle (Exam 2, 2008-2011, $N=1522$ ). This study was approved under Boston University Medical Center protocol H-27984. Written informed consent was obtained from each participant.}

\section{DNA methylation profiling and data normalization. DNA samples were} extracted from whole blood buffy coat samples using the Gentra Puregene DNA extraction kit (Qiagen, Venlo, Netherland) and subsequently underwent bisulfite conversion using the EZ DNA methylation kit (Zymo Research, Irvine, CA). Samples underwent whole-genome amplification, fragmentation, array hybridization, and single-base pair extension. DNA methylation levels were measured using the Illumina Infinium Human Methylation450 BeadChip (450 K). FHS offspring cohort samples were run in two laboratory batches at the Johns Hopkins Center for Inherited Disease Research (lab batch \#1) and University of Minnesota Biomedical Genomics Center (lab batch \#2). DNA methylation arrays of the FHS Third Generation cohort samples (lab batch \#3) were run by Illumina (San Diego, CA, USA).

For each lab batch, DNAm $\beta$ were normalized using the DASEN methodology implemented in the wateRmelon $\mathrm{R}$ package, and the output $\beta$ values for each $\mathrm{CpG}$ were used in downstream analysis ${ }^{48}$. For sample quality control, we excluded (1) samples with missing methylation values (detection $P>0.01$ ) at $>1 \% \mathrm{CpGs}$, (2) samples with poor matching between the 65 single nucleotide polymorphisms (SNPs) on the Illumina $450 \mathrm{~K}$ array and the GWAS array, and 3) samples containing outliers at the multi-dimensional scaling plot. A total of 4170 samples passed final QC, including 2648 Offspring Generation cohort samples and 1522 Third Generation cohort samples. For QC at the probe level, we excluded probes with missing methylation values (detection $P>0.01$ ) at $>20 \%$ samples, probes previously identified to map to multiple locations ${ }^{49}$ on sex chromosomes, and probes with an underlying SNP (minor allele frequency [MAF] $>5 \%$ in EA 1000 Genomes Project data) at the CpG site or within $10 \mathrm{bp}$ of the single-base extension ${ }^{50}$. A total of $415,318 \mathrm{CpGs}$ were retained for further analysis.

Genotyping and genotype imputation. Genotyping was performed using the Affymetrix $500 \mathrm{~K}$ mapping array and the Affymetrix $50 \mathrm{~K}$ gene-focused MIP array Quality control was conducted as described previously ${ }^{51}$. Genotypes were imputed from the 1000 Genomes Project panel phase 2 consisting of approximately 36.3 million SNPs using MACH / Minimac software ${ }^{52}$. SNPs with MAF $>0.01$ and imputation quality ratio $>0.3$ were retained, resulting in approximately 8.5 million SNPs that were used for further meQTL mapping.

meQTL mapping. Because of the computational burden of running linear mixed effects (LME) models for 8.5 million SNPs $\times 415 \mathrm{~K} \mathrm{CpGs,} \mathrm{we} \mathrm{adapted} \mathrm{a} \mathrm{two-step}$ analysis strategy. Step one consisted of pre-adjusting the normalized DNAm $\beta$ values for age, sex, top 50 methylation principle components (PC), predicted blood cell fraction $^{53}$, and pedigree by LME implemented in the R package Pedegreemm ${ }^{54}$ from which the resulting residuals were retained. Then, linear regression was implemented using a Java script to test the associations between DNAm residuals and the imputed SNP dosage. The top 50 methylation PCs were chosen to maximize the internal replication rate across discovery-validation split samples (50-50\%) in FHS. SNP-DNAm pairs residing within $1 \mathrm{Mb}($ cis $)$ and those residing more than $1 \mathrm{Mb}$ apart (trans) were identified separately. We used liberal $P$-value thresholds to pre-filter the meQTLs at $P<1 \mathrm{E}-6$ for cis and $P<1 \mathrm{E}-10$ for trans. Step two consisted of using the LME model implemented in the lmekin() function from the kinship2 $\mathrm{R}$ package $\mathrm{e}^{55}$ to re-calculate the associations between SNPs and DNAm for the pre-selected SNP-DNAm pairs by modeling the SNP as the independent variable, DNAm as the outcome, and adjusting for the same covariates as in step 1 as well as familial relatedness. The coefficients of co-ancestry were 0.5 between parent and offspring, 0.5 between siblings, and 0 between all other individuals. Bonferroni correction was used to maintain an experiment-wide type I error rate of 0.05 for $2.5 \times 10^{9}$ cis SNP-DNAm pairs and $(415 \mathrm{~K} \mathrm{CpGs} \times 8.5 \mathrm{M}$ SNPs $-2.5 \times 10^{9}$ cis-pairs) trans pairs, respectively, based on the CpGs and SNPs from the February 2009 assembly of the human genome (hg19, GRCh37 Genome Reference Consortium Human Reference 37). The corresponding significance thresholds are $P<2 \mathrm{E}-11\left[0.05 /\left(2.5 \times 10^{9}\right)\right]$ for cis- and $P<1.5 \mathrm{E}-14[0.05 / 3.5 \times$ $\left.10^{12}\right]$ for trans-meQTLs.

The estimates of $h_{\text {cis-meQTLs }}^{2}$ (i.e. the proportion of the total variance in a CpG explained by all of its cis-meQTL variants) and $h_{\text {trans-meQTLs }}^{2}$ (i.e. the proportion of the total variance in a CpG explained by all of its trans-meQTL variants) were calculated using 779 independent individuals by selecting one individual from each family in the FHS. To reduce the computational burden, we only used cis- and trans-meQTL SNPs that were genotyped. $h_{\text {cis-meQTLs }}^{2}$ and $h_{\text {trans-meQTLs }}^{2}$ were calculated using the GREML function in the GCTA package ${ }^{56}$, adjusting for age, sex, top 50 methylation PCs and predicted blood cell fraction.

Determination of the genomic control factor. The genomic control factor $(\lambda)$ was defined previously ${ }^{57}$. In our study, due to extensive storage requirements (i.e., storage of at least half of the raw results) to compute $\lambda$, we computed $\lambda$ based on associations of $415 \mathrm{~K} \mathrm{CpGs}$ with a random subsample of 100,000 SNPs selected from the 1000 Genomes Project panel with MAF $>0.01$ and imputation quality ratio $>0.3$ and also within the HapMap2 SNP set.

mRNA expression data. Whole blood samples $(2.5 \mathrm{ml})$ were collected in PAX gene $^{\text {mi }}$ tubes (PreAnalytiX, Hombrechtikon, Switzerland). mRNA expression was profiled using the Affymetrix Human Exon 1.0 ST GeneChip platform. Raw gene expression data were first normalized using the RMA (robust multi-array average) from Affymetrix Power Tools (APT, thermofisher.com/us/en/home/life-science/ microarray-analysis/affymetrix.html\#1_2) with quantile normalization. Then output expression values of 17,318 genes were extracted by APT based on NetAffx annotation version $31^{58}$. The gene expression values were adjusted for a set of technical covariates, e.g. chip batch, by fitting LME models as described previously ${ }^{59}$. mRNA expression data were available for 5626 individuals from the FHS Offspring cohort (Exam 8, N=2446) and Third Generation cohort (Exam 2, $N=$ 3180).

Estimating the heritability of DNAm. The narrow-sense heritability, estimated for the methylation level of individual CpGs (denoted as $h_{C p G}^{2}$ ), was the proportion of total phenotypic genetic variance $\left(\sigma_{C p G}^{2}\right)$ due to the additive polygenic genetic 
variance $\left(\sigma_{A}^{2}\right): h_{C p G}^{2}=\sigma_{A}^{2} / \sigma_{C p G}^{2} . \sigma_{C p G}^{2}=\sigma_{A}^{2}+\sigma_{h}^{2}+\sigma_{e}^{2}$, where $\sigma_{h}^{2}$ is the household variance and $\sigma_{e}^{2}$ is the environmental variance. LME regression models (using the lmekin() function of the kinship2 R Package ${ }^{55}$ ) were used to estimate $h_{C p G}^{2}$ by modeling methylation values from the familial relatedness matrix with age, sex, top 50 methylation PCs, cell type fractions, and a household matrix as covariates. The coefficients of co-ancestry in the familial relatedness matrix are 0.5 between parent and offspring, 0.5 between siblings, and 0 between all other individuals. The household effect defined as the proportion of variances in a $\mathrm{CpG}$ was attributed to the individuals sharing household. The coefficient of shared household is 1 between individuals in the same family, and 0 between all other individuals.

MR tests for the relations of DNAm and CVD. Two-sample Mendelian randomization (MR) was used to identify putatively causal CpGs for CVD and its risk factors using a multi-step strategy. Estimated associations and effect sizes between SNPs and traits were based on the latest published meta-analysis GWAS of coronary heart disease $(\mathrm{CHD})^{21}$; myocardial infarction (MI ${ }^{21}$; type-II diabetes (T2D) ${ }^{22}$; body mass index (BMI) ${ }^{23}$; lipids traits including high-density lipoprotein (HDL) cholesterol, low-density lipoprotein (LDL) cholesterol, total cholesterol (TC), and triglycerides $(\mathrm{TG})^{24}$; systolic blood pressure (SBP); and diastolic blood pressure (DBP ${ }^{25}$. Instrumental variables (IVs) for each CpG site were composed of independent cismeQTLs pruned at LD $r^{2}<0.01$, retaining only the cis-meQTL variant with the lowest SNP-CpG $P$-value in each LD block. The LD proxies were defined using 1000 genomes European samples ${ }^{60}$. Inverse variance weighted (IVW) MR tests were performed on CpGs with at least three independent cis-meQTL variants, which is the minimum number of IVs needed to perform multi-instrument MR. For CHD, MI, SBP, DBP, and T2D GWAS results based on 1000 Genome Project reference panels, MR tests were performed on 14,910 CpGs. For lipid traits and BMI, with GWAS results based on the Metabochip genotyping platform, MR tests were performed on 9,921 CpGs, with at least 3 independent cis-meQTLs on the Metabochip. A large proportion of meQTLs were not measured on the Metabochip genotyping platform, which limits MR analyses for BMI and lipid traits. When the manuscript was under preparation, a new GWAS for CHD was published that was not incorporated in the MR analysis ${ }^{61}$

To test the validity of IVW-MR results, we performed heterogeneity and MREGGER pleiotropy tests for all IVs ${ }^{62}$. We used a step-wise strategy to select valid IVs for MR. If either $P_{\text {heter }}$ or $P_{\text {pleio }}$ were less than 0.05, we excluded the top outlier IV. IVW-MR, heterogeneity, and pleiotropy tests were repeated using the remaining IVs. Finally, significant causal CpGs for a trait were required to meet the following restrictions: (1) have at least 3 independent SNPs, (2) Bonferronicorrected $P_{M R}<0.05$, corrected for 14,910 for CHD, MI, SBP, DBP, and T2D, or Bonferroni-corrected $P_{M R}<0.05$, corrected for 9,921 for lipids traits and BMI, and 3) both $P_{\text {heter }}$ and $P_{\text {pleio }}>0.05$.

Because the cis-meQTLs were identified using a $2 \mathrm{Mb}$ window, it is possible that CpGs located within $2 \mathrm{Mb}$ shared cis-meQTLs (IVs). CpGs located in close proximity may be highly correlated and involved in the similar biological pathways. The remaining $\mathrm{CpGs}$, although partially influenced by same set of genetic variants, exhibited only moderate correlation $\left(r^{2}<0.5\right)$, and should be considered as independent epigenetic loci, raising the question of "horizontal pleiotropy IVs" for traditional MR analysis. To overcome this problem, we further applied multivariable MR methods ${ }^{63}$ to moderately correlated CpGs located within $2 \mathrm{Mb}$ of each other, and tested against the same outcome to simultaneously estimate the causal effect of each $\mathrm{CpG}$ on the outcome. The multivariable MR method is analogous to the simultaneous assessment of several treatments in a factorial randomized trial, and was successfully applied to simultaneously estimate causal effects of different lipid fractions on $\mathrm{CVD}^{63}$. MR analyses were conducted using the MRbase package in $\mathrm{R}^{64}$.

Identification of associations of gene expression and DNAm. Association tests of DNAm and gene expression were performed in 3684 FHS participants with available DNAm and mRNA data. DNAm $\beta$ values were adjusted for age, sex, predicted blood cell fraction, top 2 PCs of DNAm, and 25 surrogate variables (SVs), with DNAm as fixed effects, and batch as random effects by fitting LME models. Residuals (DNAm_resid) were retained. The gene expression values (RMA, see "mRNA expression data" section) were adjusted for age, sex, predicted blood cell fraction, a set of technical covariates ${ }^{59}$, the two top PCs and 25 SVs, with gene expression as fixed effects, and batch as random effects by LME, and residuals (mRNA_resid) were retained. Then, linear regression models were used to assess pair-wise associations between DNAm_resid and mRNA_resid. SVs were calculated using the SVA package in $\mathrm{R}^{65}$. The significant threshold is Bonferronicorrected $P<0.05\left(0.05 /\left[415 \mathrm{~K} \mathrm{CpGs} \times 18 \mathrm{~K}\right.\right.$ mRNAs] $\left.=6.2 \times 10^{-12}\right)$. A cis-CpGmRNA pair was defined as a $\mathrm{CpG}$ residing $\pm 1 \mathrm{Mb}$ of the TSS of the corresponding gene encoding the mRNA (cis-eQTM). The annotations of CpGs and transcripts were obtained from annotation files of the HumanMethylation450K BeadChip and the Affymetrix exon array S1.0 platforms.

Colocalization analysis. Colocalization analysis was performed on cis-CpGmRNA pairs (see Identification of associations of gene expression and DNAm) for the 92 putatively causal CpGs for CVD traits identified by MR (see Mendelian randomization test for relationships of $\mathrm{CpGs}$ and $\mathrm{CVD}$ phenotypes). Colocalization tests were performed on each cis-CpG-mRNA pair using corresponding cis-meQTL and cis-eQTL variants. A Bayesian colocalization method implemented in the coloc $\mathrm{R}$ package was used to test the probability of one distinct variant being causal for both the $\mathrm{CpG}$ and gene expression ${ }^{66}$, by assigning each cis-SNP-CpG-mRNA pair to one of the five hypothesis. $\mathrm{H} 0$ : there exist no causal variants for either the $\mathrm{CpG}$ or the mRNA; $\mathrm{H} 1$ : there exists a causal variant for the $\mathrm{CpG}$ only; $\mathrm{H2}$ : there exists a causal variant for the mRNA only; $\mathrm{H} 3$ : there exist two distinct causal variants, one for the $\mathrm{CpG}$ and one for the mRNA; or H4: there exists a single causal variant for both the CpG and the mRNA. The result of this procedure is five posterior probabilities (PP0, PP1, PP2, PP3 and PP4) for each hypothesis. In this study, the association results between SNPs and DNAm (at $P<1 \mathrm{E}-6)$, and between SNPs and gene expression (at $P<1 \mathrm{E}-4$ ) within a $2 \mathrm{Mb}$ region were used as input. Prior probabilities were set as suggested by previous studies 18,67 -the probability of a SNP being associated with trait 1 only $(\mathrm{p} 1)$ was $2 \mathrm{E}-11$, the probability of a SNP being associated with trait 2 only (p2) was $1 \mathrm{E}-7$, the probability of a SNP being associated with both traits (p12) was p1 multiplied by $10 \%$. The $\mathrm{p} 12=\mathrm{p} 1 \times 10 \%$ indicates the probability of a causal SNP for DNAm also being the causal SNP for gene expression at $10 \%$. Those parameters revealed almost the same results when $\mathrm{p} 1$ and $\mathrm{p} 2$ are both set to $1 \mathrm{E}-4$, and $\mathrm{p} 12$ is set to $1 \mathrm{E}-5$. A SNP was considered to be colocalized for $\mathrm{CpG}$ and mRNA if the posterior probability (PP4) was greater than or equal to $80 \%$.

MR tests for the relations of gene expression to CVD. To evaluate if gene expression is causal for CVD phenotypes in whole blood, independent cis-eQTLs (pruned by LD $r^{2}<0.01, n \geq 3$ ) form FHS eQTL resource ${ }^{16}$ were used as IVs and IVW-MR was performed to test if changes in gene expression levels were causal for CVD phenotypes. To carefully select IVs unconfounded by heterogeneity and pleiotropy, we used the same strategy as described in the Mendelian randomization test for the relationships between CpG and CVD phenotypes section.

In order to test if gene expression in other tissues is causal for CVD phenotypes, we used cis-eQTL variants identified from 44 tissues by GTEx ${ }^{26}$ as IVs. IVW-MR tests were used in relation to gene expression when more than three independent cis-eQTL variants (LD $\left.r^{2}<0.01\right)$ were available. If there were fewer than three independent cis-eQTL variants for gene expression available, we used the top ciseQTL variant as an IV for MR testing. The statistical significance threshold was $P_{M R}<0.05 / 8$

Functional annotation of CpGs and meQTLs. Mapping and annotation of CpGs on the HumanMethylation450K BeadChip has been described previously 49 Genomic features of CpGs were annotated, including CpGs located in CpG Islands, low or high $\mathrm{CpG}$ regions, promoter, enhancer, gene body, 3 prime untranslated region (3'UTR), 5'UTR, 0-200 bases upstream of transcription start sites (TSS200), and TSS1500. Hypergeometric tests were used to evaluate if the identified cis- and trans-meQTL CpGs showed enrichment for CpGs annotated with those genomic features. The significance threshold was defined by a fold change of $>1.2$ or $<0.8$ and a Bonferroni-corrected $P<0.05 / 10$

Epigenome Roadmap Project data ${ }^{15}$ were used to determine whether the detected cis- and trans-meQTL SNPs were enriched for functional regions in the genome. We used data from primary cell lines of peripheral blood, including E029 monocytes, E032 B-cells, E034 T-cells, E037 T-helper memory cells, E038 T-helper naive cells, E039 T-helper naive cells, E040 T-helper memory cells, E043 T-helper cells, E044 T-regulatory cells, E045 primary T-cells effector/memory enriched, E046 natural killer cells, E047 T-CD8 + naive cells, E048 T-CD8+ memory cells, and E062 mononuclear cells, and from other CVD relevant tissues including E063 adipose nuclei, E065 aorta, E066 liver, E067 brain angular gyrus, E068 brain anterior caudate, E069 brain cingulate gyrus, E071 brain hippocampus middle, E072 brain inferior temporal lobe,E073 brain dorsolateral prefrontal cortex, E074 brain substantia nigra, E087 pancreatic islets, E095 left ventricle, E096 lung, E098 pancreas, E100 psoas muscle, E104 right atrium, E105 right ventricle, and E108 skeletal muscle. Eighteen chromatin states included active transcription start site (TssA), flanking transcription start site (TssFlnk), upstream flanking transcription start site (TssFlnkU), downstream flanking transcription start site (TssFlnkD), strong transcription (Tx), weak transcription (TxWk), genic enhancer (EnhG1 and EnhG2), active enhancer (EnhA1 and EnhA2), weak enhancer (EnhWk), zinc finger genes and repeats (ZNF_Rpts), heterochromatin (Het), bivalent/poised transcription start site (TssBiv), bivalent enhancer (EnhBiv), repressed polycomb (RepPC), weak repressed polycomb (RepPCWk), and quiescent (Quies).

To test whether the detected cis- and trans-meQTLs SNPs were enriched for SNPs residing in genomic regions annotated with chromatin states ("chromatin states SNPs"), we used a permutation-based strategy by randomly selecting equal numbers of MAF-matched SNPs 1000 times. Fold change was calculated as the ratio of the overlap between the tested-SNPs (i.e., cis- or trans-meQTL) and chromatin states SNPs to the overlap between the permutation-SNPs and chromatin states SNPs. The pools of candidate SNPs were from 1000-genomes imputed SNPs with MAF $>0.01$ and imputation quality ratio $>0.3$ as described above. To match the distribution of MAFs of the permutation-SNP set with the tested-SNP set, we categorized MAF into four categories: MAF of $(0.01,0.05)$, $(0.05,0.1),(0.1,0.2)$, and $(0.2,0.5)$. For each MAF category, we kept the proportion of SNPs in the permutation SNP set equal to the proportion of SNPs in the tested 
SNP set. For cis-meQTLs, the proportions of SNPs in the four MAF categories were $18,14,21$, and $47 \%$, respectively. For trans-meQTLs, the proportions of SNPs in the four MAF categories were 12,12, 21, and $55 \%$, respectively. The statistical significance threshold was permutation-based $\mathrm{FDR}<0.05$ from 1000 permutation.

Pathway analysis. To investigate possible pathways underling the associations between meQTLs and CVD traits, we used FUMA (Functional mapping and annotation of GWAS $)^{27}$ on the cis-meQTLs for the putatively causal CpGs identified by MR analysis. FUMA included all loci with cis-meQTLs at LD $>0.8$ based on the 1000 Genomes references panel as input. Hypergeometric tests on genes from those loci were used to investigate over representations of genes from multiple pathways. To improve focus in this study, we only use results of $\mathrm{KEGG}^{68}$ and Gene Ontology-biological process (GO-BP) terms ${ }^{69}$. The SNP-to-Gene mapping was used on associations between SNPs and genes from positional mapping in Grch37/hg19, eQTLs in GTEx ${ }^{26}$, and chromatin interaction mapping in Hi-C databases ${ }^{70}$. The significant threshold for the pathway analysis used a corrected $P<$ 0.05/tests pathways in FUMA.

Replication of cis- and trans-meQTLs in independent studies. For the significant cis- and trans-meQTLs identified in FHS, we attempted replication of the SNP-CpG pairs in 963 ARIC EA participants and 384 GTP AA cohort participants. Due to lower numbers of samples in these cohorts, we excluded SNPs with MAF < 0.05 in ARIC and GTP.

ARIC Study is a prospective cohort conducted in four US communities to investigate atherosclerosis and clinical atherosclerotic diseases ${ }^{71}$. A total of 15,792 men and women aged 45 to 64 years (baseline) were recruited in 1987 and 1989 (visit 1) from four communities: Forsyth County, North Carolina; Jackson, Mississippi; suburbs of Minneapolis, Minnesota; and Washington County, Maryland. The ARIC study protocol was approved by the institutional review board of each participating university. Four subsequent follow-up exams were carried out in 1990-1992 (visit 2), 1993-1995 (visit 3), 1996-1998 (visit 4), and 2011-2013 (visit 5). DNA methylation was measured for samples from visit 2 or visit3. Genomic DNA was extracted from peripheral whole blood samples using the Gentra Puregene Blood Kit (Qiagen; Valencia, CA, USA). Bisulfite-conversion of DNA samples was performed using the EZ-96 DNA Methylation Kit (Deep Well Format) (Zymo Research; Irvine, CA, USA), and then measured for methylation status using the Illumina HumanMethylation $450 \mathrm{~K}$ beadarray (Illumina, Inc., San Diego, CA, USA). Probe intensities were extracted using Illumina GenomeStudio software (version 2011.1, Methylation module 1.9.0). Poor-quality samples with pass rate less than $95 \%$ were excluded. Samples were further excluded based on gender mismatch, SNP discordance with previous genotyping, and outliers in PC analysis. At the target level, poor-quality CpG sites missing in $\geq 5 \%$ samples were excluded. Beta values, representative of the methylation score for each $\mathrm{CpG}$, were normalized using the Beta MIxture Quantile dilation (BMIQ) $\operatorname{method}^{72}$. Blood cell types were imputed using the Houseman method $^{73}$. Genotyping was performed with Affymetrix SNP array 6.0. Imputation of missing genotypes was performed using IMPUTE2 $2^{74}$ using $1000 \mathrm{G}$ Phase 1 version 3 reference. We first fit LME models, where the BMIQ normalized beta values were the response variables, the covariates age, sex, visit number, blood cell counts, and the top 50 methylation PCs were fitted via fixed effects, and the batch factors chip ID, chip position, plate number were fitted as random effects. Residuals extracted from the fitted model were tested for associations with SNPs using linear regression models. All analysis were carried out in $\mathrm{R}$.

GTP is a population-based prospective study to assess trauma exposure and stress-related outcomes in an urban, predominantly AA population ${ }^{75}$. Participants were recruited prospectively from the waiting rooms of primary care and obstetrics-gynecology clinics of Grady Memorial Hospital in Atlanta, GA. Since its inception in 2005, over 5000 participants have been interviewed for the study; 384 AA participants had both genotype data and whole blood DNA methylation measurements. Genotyping and DNA methylation profiling of GTP samples are described in the previous study ${ }^{76}$. Missing data points were defined by (1) a detection p-value greater than 0.001 , or (2) a combined signal less than $25 \%$ of the total median signal and less than both the median unmethylated and median methylated signal. Individual samples were removed if they were outliers using a hierarchical clustering analysis, or had (1) a mean total signal less than half of the median of the overall mean signal or 2000 arbitrary units and (2) a missingness rate above $5 \%$. Similarly, $\mathrm{CpG}$ probes were removed if the missingness rate was above $10 \%$ or the probes overlapped with SNPs (for Infinium I probes, SNPs at the site of single-base extension; for Infinium II probes, SNPs at CpG site; for both probes, SNPs located within 10 bp from site of single-base extension) with MAF $>0.05$ in 1000 Genomes Project 20110521 release for African population (AFR) ${ }^{50}$. To remove the effect of outliers and ensure normality, DNAm $\beta$ values were first inverse-normal transformed before analysis ${ }^{77}$. Associations between 437,229 CpGs and 9,892,561 SNPs (imputed allele dosage) were then estimated using linear regression adjusting age, gender and top $20 \mathrm{PCs}$ of DNAm. The number of DNAm PCs was determined to achieve the highest power for cis-meQTLs mapped by at least one SNP $\left(P<10^{-6}\right.$, cis-meQTL was defined as CpG-SNP distance $\left.<1 \mathrm{Mb}\right)$. All meQTL analyses were performed using the R package MatrixEQTL ${ }^{78}$. Associations between cis- and trans- SNP-DNAm pairs were identified and reported separately. The Institutional Review Boards of Emory University School of Medicine and
Grady Memorial Hospital approved the study protocol for the Grady Trauma Project.

We calculated the ratio of meQTLs replicated in ARIC and GTP at $P<0.05, P<$ 0.001 , and at Bonferroni-corrected $P<0.05$, corrected for $26.8 \mathrm{M}$ tests for $c i s$ and 2 $\mathrm{M}$ tests for trans. We also compared the T-values of SNP-CpG pairs identified in FHS with T-values in ARIC and GTP.

Data resources for eQTLs and pQTLs. To examine the overlap of meQTLs with eQTLs and PQTLs, we used published resources of whole blood eQTLs identified in the FHS ${ }^{16}$ and in a prior meta-analysis of European cohorts ${ }^{17}$. Plasma pQTLs were previously identified in the $\mathrm{FHS}^{18}$ and KORA ${ }^{19}$.

Reporting summary. Further information on research design is available in the Nature Research Reporting Summary linked to this article.

\section{Data availability}

The complete set of DNAm data and mRNA expression data for FHS participants have been deposited in and are available from dbGaP under the study accession phs000724.v7. p11 (DNAm data) (https://www.ncbi.nlm.nih.gov/projects/gap/cgi-bin/study.cgi? study_id=phs000724.v7.p11) and phs000363.v17.p11 (mRNA expression data) (https:// www.ncbi.nlm.nih.gov/projects/gap/cgi-bin/study.cgi?study_id=phs000363.v17.p11). The meQTL resources developed for this study are freely accessible via the NCBI Molecular QTL Browser (https://preview.ncbi.nlm.nih.gov/gap/eqtl/studies/) and via the NCBI ftp site (https://ftp.ncbi.nlm.nih.gov/eqtl/original_submissions/FHS_meQTLs/).

Received: 20 December 2018 Accepted: 23 July 2019

Published online: 19 September 2019

\section{References}

1. Jones, P. A. \& Takai, D. The role of DNA methylation in mammalian epigenetics. Science 293, 1068-1070 (2001).

2. Portela, A. \& Esteller, M. Epigenetic modifications and human disease. Nat. Biotechnol. 28, 1057-1068 (2010).

3. Fraga, M. F. et al. Epigenetic differences arise during the lifetime of monozygotic twins. Proc. Natl Acad. Sci. USA 102, 10604-10609 (2005).

4. McRae, A. F. et al. Contribution of genetic variation to transgenerational inheritance of DNA methylation. Genome Biol. 15, R73 (2014).

5. Horvath, S. DNA methylation age of human tissues and cell types. Genome Biol. 14, 3156 (2013).

6. Joehanes, R. et al. Epigenetic signatures of cigarette smoking. Circ. Cardiovasc. Genet. 9, 436-447 (2016).

7. Liu, C. et al. A DNA methylation biomarker of alcohol consumption. Mol. Psychiatry 23, 422 (2018)

8. Wahl, S. et al. Epigenome-wide association study of body mass index, and the adverse outcomes of adiposity. Nature 541, 81-86 (2017).

9. Dekkers, K. F. et al. Blood lipids influence DNA methylation in circulating cells. Genome Biol. 17, 138 (2016).

10. Lemire, $M$. et al. Long-range epigenetic regulation is conferred by genetic variation located at thousands of independent loci. Nat. Commun. 6, 6326 (2015).

11. Bonder, M. J. et al. Disease variants alter transcription factor levels and methylation of their binding sites. Nat. Genet. 49, 131 (2017).

12. Shi, J. et al. Characterizing the genetic basis of methylome diversity in histologically normal human lung tissue. Nat. Commun. 6, 3365 (2014).

13. Gaunt, T. R. et al. Systematic identification of genetic influences on methylation across the human life course. Genome Biol. 17, 61 (2016).

14. McClay, J. L. et al. High density methylation QTL analysis in human blood via next-generation sequencing of the methylated genomic DNA fraction. Genome Biol. 16, 291 (2015).

15. Kundaje, A. et al. Integrative analysis of 111 reference human epigenomes. Nature 518, 317-330 (2015)

16. Joehanes, R. et al. Integrated genome-wide analysis of expression quantitative trait loci aids interpretation of genomic association studies. Genome Biol. 18, 16 (2017).

17. Westra, H.-J. et al. Systematic identification of trans eQTLs as putative drivers of known disease associations. Nat. Genet. 45, 1238-1243 (2013).

18. Yao, C. et al. Genome-wide mapping of plasma protein QTLs identifies putatively causal genes and pathways for cardiovascular disease. Nat. Commun. 9, 3268 (2018).

19. Suhre, K. et al. Connecting genetic risk to disease end points through the human blood plasma proteome. Nat. Commun. 8, 14357 (2017).

20. MacArthur, J. et al. The new NHGRI-EBI Catalog of published genome-wide association studies (GWAS Catalog). Nucleic Acids Res. 45, D896-D901 (2017). 
21. Nikpay, M. et al. A comprehensive 1000 Genomes-based genome-wide association meta-analysis of coronary artery disease. Nat. Genet. 47, 1121 (2015).

22. Scott, R. A. et al. An expanded genome-wide association study of type 2 diabetes in Europeans. Diabetes 66, 2888-2092 (2017).

23. Locke, A. E. et al. Genetic studies of body mass index yield new insights for obesity biology. Nature 518, 197 (2015).

24. Willer, C. J. et al. Discovery and refinement of loci associated with lipid levels. Nat. Genet. 45, 1274 (2013).

25. Evangelou, E. et al. Genetic analysis of over 1 million people identifies 535 new loci associated with blood pressure traits. Nat. Genet. 50, 1412 (2018).

26. Consortium, G. Genetic effects on gene expression across human tissues. Nature 550, 204 (2017).

27. Watanabe, K., Taskesen, E., Bochoven, A. \& Posthuma, D. Functional mapping and annotation of genetic associations with FUMA. Nat. Commun. 8, 1826 (2017)

28. Bell, J. T. et al. Epigenome-wide scans identify differentially methylated regions for age and age-related phenotypes in a healthy ageing population. PLoS. Genet. 8, e1002629 (2012)

29. Kulkarni, H. et al. Novel epigenetic determinants of type 2 diabetes in Mexican-American families. Hum. Mol. Genet. 24, 5330-5344 (2015).

30. Day, K. et al. Heritable DNA methylation in CD4+ cells among complex families displays genetic and non-genetic effects. PLoS. ONE. 11, e0165488 (2016).

31. Gaziano, T. A., Bitton, A., Anand, S., Abrahams-Gessel, S. \& Murphy, A. Growing epidemic of coronary heart disease in low-and middle-income countries. Curr. Probl. Cardiol. 35, 72-115 (2010).

32. Mendelson, M. M. et al. Association of body mass index with DNA methylation and gene expression in blood cells and relations to cardiometabolic disease: a mendelian randomization approach. PLoS. Med. 14, e1002215 (2017).

33. Hedman, Å. K. et al. Epigenetic patterns in blood associated with lipid traits predict incident coronary heart disease events and are enriched for results from genome-wide association studies. Circulation: Cardiovascular Genet. 10, e001487 (2017).

34. Richard, M. A. et al. DNA methylation analysis identifies loci for blood pressure regulation. Am. J. Hum. Genet. 101, 888-902 (2017).

35. Zwamborn, R. A. et al. Prolonged high-fat diet induces gradual and fat depot-specific DNA methylation changes in adult mice. Sci. Rep. 7, 43261 (2017).

36. Richardson, T. G. et al. Mendelian randomization analysis identifies CpG sites as putative mediators for genetic influences on cardiovascular disease risk. Am. J. Hum. Genet. 101, 590-602 (2017).

37. Yatsu, F., Hagemenas, F., Manaugh, L. \& Galambos, T. Cholesteryl ester hydrolase activity in human symptomatic atherosclerosis. Lipids 15, 1019-1022 (1980).

38. Hinds, D. A. et al. Genome-wide association analysis of self-reported events in 6135 individuals and 252827 controls identifies 8 loci associated with thrombosis. Hum. Mol. Genet. 25, 1867-1874 (2016).

39. Garrison, R. et al. ABO blood group and cardiovacular disease the Framingham study. Atherosclerosis 25, 311-318 (1976).

40. Otto, E. A. et al. Candidate exome capture identifies mutation of SDCCAG8 as the cause of a retinal-renal ciliopathy. Nat. Genet. 42, 840 (2010).

41. Schaefer, E. et al. Mutations in SDCCAG8/NPHP10 cause Bardet-Biedl syndrome and are associated with penetrant renal disease and absent polydactyly. Mol. Syndromol. 1, 273-281 (2010).

42. Airik, R. et al. Renal-retinal ciliopathy gene Sdccag8 regulates DNA damage response signaling. J. Am. Soc. Nephrol. 25, 2573-2583 (2014).

43. Zhou, J. et al. Tissue-specific DNA methylation is conserved across human, mouse, and rat, and driven by primary sequence conservation. BMC Genomics 18, 724 (2017).

44. Yao, C. et al. Dynamic role of trans regulation of gene expression in relation to complex traits. Am. J. Hum. Genet. 100, 571-580 (2017).

45. Dawber, T. R., Meadors, G. F. \& Moore, F. E. Jr Epidemiological approaches to heart disease: the Framingham Study. Am. J. Public Health Nations Health 41, 279-286 (1951).

46. Feinleib, M., Kannel, W. B., Garrison, R. J., McNamara, P. M. \& Castelli, W. P. The Framingham offspring study. Design and preliminary data. Prev. Med. 4, 518-525 (1975).

47. Splansky, G. L. et al. The third generation cohort of the National Heart, Lung, and Blood Institute's Framingham Heart Study: design, recruitment, and initial examination. Am. J. Epidemiol. 165, 1328-1335 (2007).

48. Pidsley, R. et al. A data-driven approach to preprocessing Illumina $450 \mathrm{~K}$ methylation array data. BMC Genomics 14, 293 (2013).

49. Price, E. M. et al. Additional annotation enhances potential for biologicallyrelevant analysis of the Illumina Infinium HumanMethylation450 BeadChip array. Epigenetics chromatin 6, 4 (2013).
50. Chen, Y.-a et al. Discovery of cross-reactive probes and polymorphic CpGs in the Illumina Infinium HumanMethylation 450 microarray. Epigenetics 8 203-209 (2013).

51. Levy, D. et al. Genome-wide association study of blood pressure and hypertension. Nat. Genet. 41, 677-687 (2009).

52. Li, Y., Willer, C. J., Ding, J., Scheet, P. \& Abecasis, G. R. MaCH: using sequence and genotype data to estimate haplotypes and unobserved genotypes. Genet. Epidemiol. 34, 816-834 (2010).

53. Houseman, E. A., Molitor, J. \& Marsit, C. J. Reference-free cell mixture adjustments in analysis of DNA methylation data. Bioinformatics 30, 1431-1439 (2014)

54. Vazquez, A., Bates, D., Rosa, G., Gianola, D. \& Weigel, K. An R package for fitting generalized linear mixed models in animal breeding1. J. Anim. Sci. 88, 497-504 (2010).

55. Sinnwell, J. P., Therneau, T. M. \& Schaid, D. J. The kinship2 R package for pedigree data. Hum. Hered. 78, 91-93 (2014).

56. Wood, A. R. et al. Defining the role of common variation in the genomic and biological architecture of adult human height. Nat. Genet. 46, 1173 (2014).

57. Devlin, B. \& Roeder, K. Genomic control for association studies. Biometrics 55, 997-1004 (1999).

58. Pruitt, K. D. et al. RefSeq: an update on mammalian reference sequences. Nucleic Acids Res. 42, D756-D763 (2013).

59. Huan, T. et al. Integrative network analysis reveals molecular mechanisms of blood pressure regulation. Mol. Syst. Biol. 11, 799 (2015).

60. Zheng, J. et al. LD Hub: a centralized database and web interface to perform LD score regression that maximizes the potential of summary level GWAS data for SNP heritability and genetic correlation analysis. Bioinformatics 33, 272-279 (2017).

61. van der Harst, P. \& Verweij, N. Identification of 64 novel genetic loci provides an expanded view on the genetic architecture of coronary artery disease. Circ. Res. 122, 433-443 (2018).

62. Bowden, J., Davey Smith, G. \& Burgess, S. Mendelian randomization with invalid instruments: effect estimation and bias detection through Egger regression. Int. J. Epidemiol. 44, 512-525 (2015).

63. Burgess, S. \& Thompson, S. G. Multivariable Mendelian randomization: the use of pleiotropic genetic variants to estimate causal effects. Am. J. Epidemiol. 181, 251-260 (2015).

64. Hemani, G. et al. The MR-Base platform supports systematic causal inference across the human phenome. elife 7, e34408 (2018).

65. Leek, J. T., Johnson, W. E., Parker, H. S., Jaffe, A. E. \& Storey, J. D. The sva package for removing batch effects and other unwanted variation in highthroughput experiments. Bioinformatics 28, 882-883 (2012).

66. Giambartolomei, C. et al. Bayesian test for colocalisation between pairs of genetic association studies using summary statistics. PLoS. Genet. 10, e1004383 (2014).

67. Pierce, B. L. et al. Co-occurring expression and methylation QTLs allow detection of common causal variants and shared biological mechanisms. Nat. Commun. 9, 804 (2018).

68. Kanehisa, M. \& Goto, S. KEGG: kyoto encyclopedia of genes and genomes. Nucleic Acids Res. 28, 27-30 (2000).

69. Ashburner, M. et al. Gene ontology: tool for the unification of biology. Nat. Genet. 25, 25 (2000).

70. Lieberman-Aiden, E. et al. Comprehensive mapping of long-range interactions reveals folding principles of the human genome. Science 326, 289-293 (2009).

71. The ARIC investigators. The Atherosclerosis Risk in Communities (ARIC) Study: design and objectives. Am. J. Epidemiol. 129, 687-702 (1989).

72. Teschendorff, A. E. et al. A beta-mixture quantile normalization method for correcting probe design bias in Illumina Infinium $450 \mathrm{k}$ DNA methylation data. Bioinformatics 29, 189-196 (2013).

73. Houseman, E. A. et al. DNA methylation arrays as surrogate measures of cell mixture distribution. BMC Bioinforma. 13, 86 (2012).

74. Howie, B. N., Donnelly, P. \& Marchini, J. A flexible and accurate genotype imputation method for the next generation of genome-wide association studies. PLoS. Genet. 5, e1000529 (2009).

75. Gillespie, C. F. et al. Trauma exposure and stress-related disorders in inner city primary care patients. Gen. Hosp. Psychiatry 31, 505-514 (2009).

76. Barfield, R. T. et al. Accounting for population stratification in DNA methylation studies. Genet. Epidemiol. 38, 231-241 (2014).

77. Aulchenko, Y. S., Ripke, S., Isaacs, A. \& Van Duijn, C. M. GenABEL: an R library for genome-wide association analysis. Bioinformatics 23, 1294-1296 (2007).

78. Shabalin, A. A. Matrix eQTL: ultra fast eQTL analysis via large matrix operations. Bioinformatics 28, 1353-1358 (2012).

\section{Acknowledgements}

The Framingham Heart Study is funded by National Institutes of Health contract N01-HC-25195 and HHSN268201500001I. The laboratory work for this investigation 
was funded by the Division of Intramural Research, National Heart, Lung, and Blood Institute, National Institutes of Health. The analytical component of this project was funded by the Division of Intramural Research, National Heart, Lung, and Blood Institute, and the Center for Information Technology, National Institutes of Health, Bethesda, MD. M.M.M. is supported by a NHLBI K99HL136875. The Atherosclerosis Risk in Communities study has been funded in whole or in part with Federal funds from the National Heart, Lung, and Blood Institute, National Institutes of Health, Department of Health and Human Services (contract numbers HHSN268201700001I, HHSN268201700002I, HHSN268201700003I, HHSN268201700004I, and HHSN268201700005I). The authors thank the staff and participants of the ARIC study for their important contributions. Funding was also supported by 5RC2HL102419 and R01NS087541. Dr. Ci Song is supported by the international postdoc fellowship award from Swedish Research Council (2016-00598). The views expressed in this manuscript are those of the authors and do not necessarily represent the views of the National Heart, Lung, and Blood Institute; the National Institutes of Health; or the U.S. Department of Health and Human Services.

\section{Author contributions}

D.L., T.H., and L.L. designed, directed, and supervised the project. T. H., L.L and D.L. drafted the papermanuscript. T.H., J.R., C.S., F.P. and Y.G. conducted the analyses. All authors participated in revising and editing the papermanuscripts. All authors have read and approved the final version of the papermanuscript.

\section{Additional information}

Supplementary Information accompanies this paper at https://doi.org/10.1038/s41467019-12228-z.
Competing interests: The authors declare no competing interests.

Reprints and permission information is available online at http://npg.nature.com/ reprintsandpermissions/

Peer review information Nature Communications thanks Johan Björkegren, Cong Guo and the other, anonymous, reviewer(s) for their contribution to the peer review of this work. Peer reviewer reports are available.

Publisher's note Springer Nature remains neutral with regard to jurisdictional claims in published maps and institutional affiliations.

(c) (i) Open Access This article is licensed under a Creative Commons Attribution 4.0 International License, which permits use, sharing, adaptation, distribution and reproduction in any medium or format, as long as you give appropriate credit to the original author(s) and the source, provide a link to the Creative Commons license, and indicate if changes were made. The images or other third party material in this article are included in the article's Creative Commons license, unless indicated otherwise in a credit line to the material. If material is not included in the article's Creative Commons license and your intended use is not permitted by statutory regulation or exceeds the permitted use, you will need to obtain permission directly from the copyright holder. To view a copy of this license, visit http://creativecommons.org/ licenses/by/4.0/.

This is a U.S. government work and not under copyright protection in the U.S.; foreign copyright protection may apply 2019 